TRANSACTIONS OF THE

AMERICAN MATHEMATICAL SOCIETY

Volume 355, Number 8, Pages 3379-3404

S 0002-9947(03)03217-3

Article electronically published on April 8, 2003

\title{
WEST'S PROBLEM ON EQUIVARIANT HYPERSPACES AND BANACH-MAZUR COMPACTA
}

\author{
SERGEY ANTONYAN
}

\begin{abstract}
Let $G$ be a compact Lie group, $X$ a metric $G$-space, and $\exp X$ the hyperspace of all nonempty compact subsets of $X$ endowed with the Hausdorff metric topology and with the induced action of $G$. We prove that the following three assertions are equivalent: (a) $X$ is locally continuum-connected (resp., connected and locally continuum-connected); (b) $\exp X$ is a $G$-ANR (resp., a $G$-AR); (c) $(\exp X) / G$ is an ANR (resp., an AR). This is applied to show that $(\exp G) / G$ is an ANR (resp., an AR) for each compact (resp., connected) Lie group $G$. If $G$ is a finite group, then $(\exp X) / G$ is a Hilbert cube whenever $X$ is a nondegenerate Peano continuum. Let $L(n)$ be the hyperspace of all centrally symmetric, compact, convex bodies $A \subset \mathbb{R}^{n}, n \geq 2$, for which the ordinary Euclidean unit ball is the ellipsoid of minimal volume containing $A$, and let $L_{0}(n)$ be the complement of the unique $O(n)$-fixed point in $L(n)$. We prove that: (1) for each closed subgroup $H \subset O(n), L_{0}(n) / H$ is a Hilbert cube manifold; (2) for each closed subgroup $K \subset O(n)$ acting non-transitively on $S^{n-1}$, the $K$-orbit space $L(n) / K$ and the $K$-fixed point set $L(n)[K]$ are Hilbert cubes. As an application we establish new topological models for tha BanachMazur compacta $L(n) / O(n)$ and prove that $L_{0}(n)$ and $\left(\exp S^{n-1}\right) \backslash\left\{S^{n-1}\right\}$ have the same $O(n)$-homotopy type.
\end{abstract}

\section{INTRODUCTION}

In 1976 J. E. West [33] asked the following question: Let $G$ be a compact, connected Lie group. Is the orbit space $(\exp G) / G$ an absolute retract, and if so, is it always homeomorphic to the Hilbert cube? In a more general form this problem appeared also in [34, Problem 1022].

These questions have remained open except when $G=S^{1}$, the circle group, where the answers are "Yes" and "No", respectively. Torunczyk and West proved in [29] that the orbit space $\left(\exp _{0} S^{1}\right) / S^{1}$ is an Eilenberg-MacLane space $\mathbf{K}(\mathbb{Q}, 2)$, where $S^{1}$ is the circle group and $\mathbb{Q}$ stands for the rationals.

Recall that if $G$ is a compact group and $X$ a metrizable $G$-space, then $\exp X$ denotes the hyperspace of all non-void compact subsets of $X$, equipped with the Hausdorff metric topology and with the induced action of $G$. We use $\exp _{0} X$ for the complement $(\exp X) \backslash\{X\}$.

Received by the editors May 1, 2000 and, in revised form, September, 15, 2002.

2000 Mathematics Subject Classification. Primary 57N20, 57S10, 54B20, 54C55, 55P91, 46B99.

Key words and phrases. Banach-Mazur compacta, G-ANR, Q-manifold, hyperspace, orbit space, homotopy type, $G$-nerve.

The author was supported in part by grant IN-105800 from PAPIIT (UNAM). 
In the first part of the present paper we give a positive answer to the first question of West's problem as a corollary to the following general result.

Theorem 1.1. Let $G$ be a compact Lie group and $X$ a metrizable $G$-space. Then the following are equivalent:

(1) $X$ is locally continuum-connected (resp., connected and locally continuumconnected),

(2) $\exp X$ is a $G$-ANR (resp., a $G$-AR),

(3) The orbit space $(\exp X) / G$ is an $A N R$ (resp., an $A R$ ).

The proof of this theorem is given in Section 3.

Since each compact Lie group $G$ is locally path-connected, Theorem 1.1, in particular, yields a positive answer to the first question of West's Problem (Corollary 3.8).

Remark 1.2. As is shown in Section 3, the implications $(1) \Longrightarrow(2) \Longrightarrow(3)$ in Theorem 1.1 are true also for arbitrary compact (not necessarily Lie) groups; $(1) \Longrightarrow(2)$ and $(1) \Longrightarrow(3)$ can be regarded as equivariant versions of Wojdyslawski's Theorem [35] as sharpened by D. Curtis [13.

In Heisey and West [17] it was proved that if $G$ is a finite group and $X$ is a nondegenerate Peano continuum, then $(\exp X) / G$ is a Hilbert cube if it is an AR. Consequently, in combination with Theorem 1.1, this implies that $(\exp X) / G$ is always a Hilbert cube whenever $X$ is a nondegenerate Peano continuum (Corollary 3.9).

The second part of this paper is devoted to the Banach-Mazur compacta.

In his 1932 book Théorie des Opérations Linéaires, S. Banach [10] introduced, for each $n \geq 2$, the space of isometry classes $[E]$ of $n$-dimensional Banach spaces equipped with the metric

$$
d([E],[F])=\ln \inf \left\{\|T\| \cdot\left\|T^{-1}\right\| \mid T: E \rightarrow F \text { is a linear isomorphism }\right\} .
$$

These spaces are now denoted by $B M(n)$ and called the Banach-Mazur compacta. The topology of these spaces continues to be of interest, and the following questions of A. Pelczyński were included in J. West's list of problems in the 1990 book Open Problems in Topology [34, Problem 899]: (1) Are the Banach-Mazur compacta $B M(n)$ AR's? (2) Are they Hilbert cubes?

The AR part of Pelczyński's problem has been solved affirmatively due to efforts of P. Fabel [16] and the author 8. While the question of whether the BanachMazur compacta $B M(n)$ are homeomorphic to the Hilbert cube remains open for all $n \geq 3$, it was answered negatively for $n=2$ in 9 .

In the second part of the paper we establish some new properties of the BanachMazur compacta and consider their relation to West's problem above. It turns out that Pelczyński's problem and West's problem are of the same nature, and both problems can be considered from a unified point of view.

We recall some necessary notation first. By $\mathcal{B}(n)$ we denote the hyperspace of all centrally symmetric (about the origin), compact, convex bodies in $\mathbb{R}^{n}$. We consider the Hausdorff metric topology on $\mathcal{B}(n)$ and the natural induced action of the full linear group $G L(n)$ on it. As usual, we shall use $O(n)$ for the orthogonal group. It is well known that $B M(n)$ is homeomorphic to the orbit space $\mathcal{B}(n) / G L(n)$ (see [34, p. 544]). 
According to a classical theorem of F. John [21], for any $A \in \mathcal{B}(n)$ there is a unique minimal-volume ellipsoid $l(A)$ containing $A$ (respectively, maximal-volume ellipsoid $j(A)$ contained in $A$ ). Usually $j(A)$ is called the John ellipsoid of $A$ and $l(A)$ is called the Löwner ellipsoid of $A$.

Let $J(n)$ and $L(n)$ be the $O(n)$-invariant subsets of $\mathcal{B}(n)$ consisting of all bodies $A \in \mathcal{B}(n)$ for which the ordinary Euclidean unit ball

$$
B^{n}=\left\{\left(x_{1}, \ldots, x_{n}\right) \in \mathbb{R}^{n} \mid x_{1}^{2}+\cdots+x_{n}^{2} \leq 1\right\}
$$

is the John ellipsoid and the Löwner ellipsoid, respectively. Some properties of these $O(n)$-spaces are studied in 9]. It was proved in 9, Theorem 4 and Remark $1]$ that $J(n)$ and $L(n)$ are global compact $O(n)$-slices for the $G L(n)$-space $\mathcal{B}(n)$. It then follows from a result of $\mathrm{H}$. Abels [1, Lemma 2.3] that $J(n)$ and $L(n)$ are $O(n)$ homeomorphic. The Banach-Mazur compactum $B M(n)$ is just the orbit space $J(n) / O(n)$ [9, Corollary 1] or, equivalently, the orbit space $L(n) / O(n)$ (see [9] Remark 1]). In what follows we shall use the model $B M(n)=L(n) / O(n)$. By $L_{0}(n)$ we denote the complement $L(n) \backslash\left\{B^{n}\right\}$, and $B M_{0}(n)=L_{0}(n) / O(n)$. As usual, we reserve the letter $Q$ for the Hilbert cube.

Here we prove four basic properties about the Banach-Mazur compacta $B M(n)$, $n \geq 2$.

Theorem 1.3. For any closed subgroup $H \subset O(n)$, the orbit space $L_{0}(n) / H$ is a $[0,1)$-stable $Q$-manifold. In particular, $B M_{0}(n)$ is a $[0,1)$-stable $Q$-manifold.

Recall that a $Q$-manifold is said to be $[0,1)$-stable if it is homeomorphic to its product with the half-open interval $[0,1)$ (see [12, Ch. V]).

Theorem 1.4. For each closed subgroup $K \subset O(n)$ acting non-transitively on $S^{n-1}$, the $K$-orbit space $L(n) / K$, as well as the $K$-fixed point set $L(n)[K]$, is a Hilbert cube. In particular, $L(n)$ is a Hilbert cube.

These theorems are proved in Section 5. However, Section 4 should also be considered as a part of those proofs, because the technique we develop there is further applied to Theorems 1.3 and 1.4 .

Remark 1.5. Below, in Lemma 7.4 the hypothesis that $K$ acts non-transitively on the sphere $S^{n-1}$ is shown to be equivalent to the condition $L_{0}(n)[K] \neq \emptyset$.

Next, in Section 6 we apply Theorem 1.3 to establish a new topological model for the Banach-Mazur compacta $B M(n)$ for arbitrary $n \geq 2$.

Namely, assume that $\left(H_{1}\right),\left(H_{2}\right), \ldots$ is the sequence of all $O(n)$-orbit types occurring in $L_{0}(n)$. Let Cone $\left(O(n) / H_{i}\right)$ denote the cone over $O(n) / H_{i}$ endowed with the quotient topology and with the translation action of $O(n)$ on the levels. Let

$$
\Pi(n)=\prod_{i=1}^{\infty} Q\left(H_{i}\right), \quad \text { where } \quad Q\left(H_{i}\right)=\left(\operatorname{Cone}\left(O(n) / H_{i}\right)\right)^{\infty},
$$

each equipped with the diagonal $O(n)$-action.

Denote $\Pi_{0}(n)=\Pi(n) \backslash\{a\}$, where $a$ is the unique $O(n)$-fixed point of $\Pi(n)$. Since Cone $\left(O(n) / H_{i}\right) \in \mathrm{AR}, i \geq 1$, it then follows from a result of West [32] that $\Pi(n)$ is a Hilbert cube.

Theorem 1.6. For each closed subgroup $H \subset O(n)$, the two $H$-orbit spaces $L_{0}(n) / H$ and $\Pi_{0}(n) / H$ are homeomorphic. In particular, the Banach-Mazur compactum $B M(n)$ is homeomorphic to the orbit space $\Pi(n) / O(n)$. 
Remark 1.7. In combination with [9, Corollary 10], Theorem[1.6 gives yet simpler topological models for $B M(2)$.

The idea that Pelczyński's problem on Banach-Mazur compacta is closely related to West's problem on equivariant hyperspaces was expressed in 9 in the following form:

Conjecture 1.8. For each closed subgroup $H \subset O(n), n \geq 2$, the orbit spaces $L_{0}(n) / H$ and $\left(\exp _{0} S^{n-1}\right) / H$ are homeomorphic $Q$-manifolds. In particular, the Banach-Mazur compactum BM(n) is homeomorphic to $\left(\exp S^{n-1}\right) / O(n)$.

Since the sphere $S^{n-1}$ is $O(n)$-homeomorphic to the coset space $O(n) / O(n-1)$, we see that $\left(\exp S^{n-1}\right) / O(n)$ is just of the form $(\exp (G / H)) / G$. So, if Conjecture 1.8 were proved, the Banach-Mazur compacta would be just of the form $(\exp (G / H)) / G$ (with $G=O(n)$ and $H=O(n-1)$ ). On the other hand, the space $(\exp G) / G$ in West's problem is also of the form $(\exp (G / H)) / G$ (with $H$ the trivial subgroup). This shows how close are, in fact, Pelczyński's problem and West's problem.

Here is our fourth result on Banach-Mazur compacta, which is proved in Section 7 :

Theorem 1.9. $\exp _{0} S^{n-1}$ and $L_{0}(n)$ have the same $O(n)$-homotopy type.

It follows immediately from Theorem 1.9 that $L_{0}(n) / H$ and $\left(\exp _{0} S^{n-1}\right) / H$ have the same homotopy type for any closed subgroup $H \subset O(n)$. This result and Theorem 1.3 constitute essential steps in proving Conjecture 1.8 . However, we do not prove Conjecture 1.8 in this paper. The only step we lack to complete its proof is that $\left(\exp _{0} S^{n-1}\right) / H, n \geq 2$, is a $Q$-manifold. The details of this reduction are also presented in Section 6 (Theorem 7.9).

The paper is divided as follows:

$\S 1$. Introduction.

§2. Preliminaries.

§3. Proof of Theorem 1.1 and its corollaries.

$\S 4$. The $G$-nerve.

$\S 5$. Proofs of Theorems 1.3 and 1.4 .

$\S 6$. Proof of Theorem 1.6

$\S 7$. Proof of Theorem 1.9] and reduction of Conjecture 1.8.

\section{Preliminaries}

For a given topological group $G$, we denote by $G$-A(N)R (resp., by $G-\mathrm{A}(\mathrm{N}) \mathrm{E}$ ) the class of all $G$-equivariant absolute (neighborhood) retracts (resp., extensors) for all metrizable $G$-spaces. These concepts are straightforward extensions to the case of $G$-spaces of the corresponding concepts of ordinary A(N)R's and A(N)E's (see, for example, 2]-[6]). We refer to the monographs [11] and [26] for basic notions of the theory of $G$-spaces.

If $G$ is a topological group and $X$ is a $G$-space, for any $x \in X$ we denote the stabilizer (or stationary subgroup) of $x$ by $G_{x}=\{g \in G \mid g x=x\}$.

For each subgroup $H \subset G$, the $H$-fixed point set $X[H]$ is defined to be the set $\left\{x \in X \mid H \subset G_{x}\right\}$.

The family of all subgroups of $G$ that are conjugate to $H$ is denoted by $(H)$, i.e., $(H)=\left\{g H g^{-1} \mid g \in G\right\}$. We will call $(H)$ a $G$-orbit type (or simply an orbit type). 
For two orbit types $\left(H_{1}\right)$ and $\left(H_{2}\right)$, one says that $\left(H_{1}\right) \preceq\left(H_{2}\right)$ iff $H_{1} \subset g^{-1} H_{2} g$ for some $g \in G$. The relation $\preceq$ is a partial ordering on the set of all orbit types. Since $G_{g x}=g G_{x} g^{-1}$ for any $x \in X$ and $g \in G$, we have $\left(G_{x}\right)=\left\{G_{g x} \mid g \in G\right\}$.

For a subset $S \subset X, H(S)$ denotes the $H$-saturation of $S$, i.e., $H(S)=\{h s \mid h \in$ $H, s \in S\}$. In particular, $H(x)$ denotes the $H$-orbit $\{h x \in X \mid h \in H\}$ of $x$. The $H$-orbit space is denoted by $X / H$. In particular, $X / G$ denotes the orbit space of $X$.

By $G / H$ we will denote the $G$-space of cosets $\{g H \mid g \in G\}$ under the action of $G$ induced by left translations.

A continuous map $f: X \rightarrow Y$ of $G$-spaces is said to be equivariant or $G$ equivariant or, for short, a $G$-map, if $f(g x)=g f(x)$ for all $g \in G, x \in X$. An equivariant map $f: X \rightarrow Y$ is said to be isovariant (or $G$-isovariant) if $G_{x}=G_{f(x)}$ for all $x \in X$.

A compatible metric $\rho$ on a $G$-space is called invariant or $G$-invariant if $\rho(g x, g y)$ $=\rho(x, y)$ for all $x, y \in X$ and $g \in G$.

If $X$ is metrized by a $G$-invariant metric $\rho$, then the formula $\widetilde{\rho}(G(x), G(y))=$ $\inf \left\{\rho\left(x^{\prime}, y^{\prime}\right) \mid x^{\prime} \in G(x), y^{\prime} \in G(y)\right\}$ defines a metric $\widetilde{\rho}$, compatible with the quotient topology of $X / G$, whenever $G$ is a compact group.

Let us recall the well-known and important definition of a slice [26]:

Definition 2.1. Let $G$ be a topological group, $H \subset G$ a closed subgroup and $X$ a $G$-space. A subset $S \subset X$ is called an $H$-slice in $X$ if

(1) $S$ is $H$-invariant, i.e., $H(S)=S$,

(2) the saturation $G(S)$ is open in $X$,

(3) if $g \in G \backslash H$, then $g S \cap S=\emptyset$, and

(4) $S$ is closed in $G(S)$.

If in addition $G(S)=X$, then we say that $S$ is a global $H$-slice of $X$.

The following is one of the fundamental results in topological transformation group theory (see [26, Corollary 1.7.19, Corollary 1.7.20 and Theorem 1.7.7] or [11, Ch. II, $§ \S 4$ and 5]):

Theorem 2.2 (Slice theorem). Let $G$ be a compact Lie group, $X$ a Tychonoff $G$-space and $x \in X$ any point. Then:

(1) There exists a $G_{x}$-slice $S \subset X$ such that $x \in S$.

(2) $\left(G_{y}\right) \preceq\left(G_{x}\right)$ for each point $y \in G(S)$.

(3) There exists a unique $G$-map $f: G(S) \rightarrow G / G_{x}$ such that $S=f^{-1}\left(e G_{x}\right)$.

In [9], using the classical result of John [21] on the minimal-volume ellipsoid, it was proved that $L(n)$ is a global $O(n)$-slice for the $G L(n)$-space $\mathcal{B}(n)$. In combination with a result of $H$. Abels [1, Theorem 2.1] this yields the following theorem, which we will need in the sequel:

Theorem 2.3. There is an $O(n)$-equivariant retraction $r: \mathcal{B}(n) \rightarrow L(n)$ such that $r(A)$ belongs to the $G L(n)$-orbit $G L(n)(A)$ for every $A \in \mathcal{B}(n)$.

In [9, Corollary 2] it was proved that $J(n)$ is a compact $O(n)$-AR, and since $L(n)$ is $O(n)$-homeomorphic to $J(n)$ 9, Remark 1], we have the following result that will often be used in what follows:

Theorem $2.4([9]) . L(n)$ is a compact $O(n)-A R$. 
Let $f_{0}, f_{1}: X \rightarrow X^{\prime}$ be $G$-maps. A $G$-homotopy of $f_{0}$ into $f_{1}$ is a homotopy in the ordinary sense which is a $G$-map at each stage of the deformation. A $G$-space $X$ is called $G$-contractible if there is a $G$-fixed point $* \in X$ such that the constant $G$-map $X \rightarrow\{*\}$ and the identity map $1_{X}$ are $G$-homotopic. A $G$-map $f: X \rightarrow Y$ is a $G$-homotopy equivalence if there is a $G$-map $f^{\prime}: Y \rightarrow X$ such that $f^{\prime} f$ is $G$-homotopic to $1_{X}$ and $f f^{\prime}$ is $G$-homotopic to $1_{Y}$.

Theorem 2.5 (20]). Let $G$ be a compact Lie group and $f: T \rightarrow Z$ a $G$-map of $G$-ANR's. Then $f$ is a G-homotopy equivalence iff for each closed subgroup $K \subset G$, the restriction of $f$ to the $K$-fixed point set $T[K]$ is an ordinary homotopy equivalence.

Remark 2.6. In 20, Proposition 4.1] the result originally was stated for paracompact $G$-ANE's (even in its fiberwise form). However, the proof in [20] serves for metrizable $G$-ANR's as well.

Yet another basic result for this paper is the following.

Theorem 2.7 ([6], 7]). Let $G$ be a compact group, $N \subset G$ a closed normal subgroup and $X$ a $G$-ANR (resp., a $G$-AR). Then the $N$-orbit space $X / N$, endowed with the induced action of the quotient group $G / N$, is a $G / N-A N R$ (resp., a $G / N-A R$ ). In particular, $X / G$ is an $A N R$ (resp., an $A R$ ).

Recall that for a metric space $(X, d)$, the Hausdorff metric $d_{H}$ on $\exp X$ is defined by the formula

$$
d_{H}(C, D)=\max \left\{\sup _{x \in D} \operatorname{dist}(x, C), \sup _{y \in C} \operatorname{dist}(y, D)\right\} \quad \text { for } \quad C, D \in \exp X .
$$

The topology generated by $d_{H}$ is an invariant of the topology of $X$ (it does not depend on $d$ ).

If $G$ is a compact group and $X$ is a metrizable $G$-space, then the formula

$$
(g, A) \longmapsto g A ; \quad g A=\{g a \mid a \in A\}, \text { for all } g \in G, A \in \exp X
$$

defines a continuous $G$-action on $\exp X$; so $\exp X$ naturally becomes a $G$-space. In this case the complement $\exp _{0} X=(\exp X) \backslash\{X\}$ is an open invariant subset of $\exp X$. Clearly, if $d$ is a $G$-invariant metric on $X$, then $d_{H}$ is a $G$-invariant metric on $\exp X$.

For the boundary of a set $A \subset X$ we will use the notation $\partial A$.

Throughout the paper we will use the following standard notation:

$$
\begin{gathered}
B^{n}=\left\{\left(x_{1}, \ldots, x_{n}\right) \in \mathbb{R}^{n} \mid x_{1}^{2}+\cdots+x_{n}^{2} \leq 1\right\}, \quad \text { the Euclidean unit ball; } \\
S^{n-1}=\left\{\left(x_{1}, \ldots, x_{n}\right) \in \mathbb{R}^{n} \mid x_{1}^{2}+\cdots+x_{n}^{2}=1\right\}, \quad \text { the Euclidean unit sphere; } \\
\Delta^{n}=\left\{\left(t_{0}, \ldots, t_{n}\right) \in \mathbb{R}^{n+1} \mid t_{i} \geq 0, t_{0}+\cdots+t_{n}=1\right\}, \text { the standard closed simplex; } \\
Q=\prod_{k=1}^{\infty}\left\{I_{k} \mid I_{k}=[0,1]\right\}, \quad \text { the Hilbert cube. }
\end{gathered}
$$




\section{Proof of Theorem 1.1 And its Corollaries}

We first prove the following equivariant version of Curtis' generalization [13, Theorem 1.6] of the well-known Wojdyslawski Theorem [35], which is just the implication $(1) \Longrightarrow(2)$ in Theorem 1.1.

Recall that a metric space $X$ is continuum-connected if each pair of points in $X$ is contained in a subcontinuum. $X$ is locally continuum-connected if it has an open base of continuum-connected subsets 13 .

Proposition 3.1. Let $G$ be a compact group and $X$ a locally continuum-connected (resp., connected and locally continuum-connected) metrizable $G$-space. Then $\exp X$ is a $G$-ANR (resp., $G$-AR).

Proof. We shall consider only the "G-ANR" case. The "G-AR" case is similar.

If $\exp X$ is a $G$-ANR, then it is also an ANR, and then by Curtis' theorem [13], $X$ is locally continuum-connected.

Now assume that $(Y, \rho)$ is a metric $G$-space with $\rho$ an invariant metric on $Y, A$ a closed invariant subset of $Y$, and $\varphi: A \rightarrow \exp X$ a $G$-map. By Curtis' theorem [13], exp $X$ is an ANR. So $f$ has a continuous extension $\varphi: U \rightarrow \exp X$ defined on a neighborhood $U$ of $A$ in $Y$. By compactness of $G$, there is an invariant neighborhood $V$ of $A$ contained in $U$. Set

$$
F(y)=\bigcup_{g \in G} g^{-1} \varphi(g y) \text { for every } y \in V .
$$

We claim that the map $F: V \rightarrow \exp X$ is a well-defined continuous $G$-equivariant extension of $f$. Indeed, for every $y \in V$ the set $\left\{g^{-1} f(g y) \mid g \in G\right\}$ is a compact subset of $\exp X$ because it is the image of the continuous map $\alpha: G \rightarrow \exp X$, $\alpha(g)=g^{-1} f(g y)$. Therefore, the union $\bigcup_{g \in G} g^{-1} \varphi(g y)$ is a compact subset of $X$, i.e., $F(y) \in \exp X$.

Further, if $t, g \in G$ and $h=g t$, then

$$
F(t y)=\bigcup_{g \in G} g^{-1} \varphi(g t y)=\bigcup_{h \in G} t\left(h^{-1} \varphi(h y)\right)=t\left(\bigcup_{h \in G} h^{-1} \varphi(h y)\right)=t F(y),
$$

showing the equivariance of $F$. If $a \in A$, then $\varphi(g a)=f(g a)$ for all $g \in G$, and by the equivariance of $f$ we will then have

$$
F(a)=\bigcup_{g \in G} g^{-1} \varphi(g a)=\bigcup_{g \in G} g^{-1} f(g a)=\bigcup_{g \in G} g^{-1} g f(a)=\bigcup_{g \in G} f(a)=f(a)
$$

showing that $F$ extends $f$.

Let $d$ be an invariant metric on $X$.

To see the continuity of $F$, we fix $y_{0} \in V$ and $\varepsilon>0$ arbitrary. By continuity of $\varphi$, for each $g \in G$ there is a $\delta_{g}>0$ such that

$$
d_{H}\left(\varphi\left(g y_{0}\right), \varphi(z)\right)<\varepsilon / 2 \text { whenever } z \in V \text { and } \rho\left(z, g y_{0}\right)<2 \delta_{g} .
$$

By compactness of the orbit $G\left(y_{0}\right)$, its open cover $\left\{O\left(g y_{0}, \delta_{g}\right) \mid g \in G\right\}$, where $O(z, r)$ stands for the open $r$-ball in $X$ centered at $z$, admits a finite subcover

$$
\left\{O\left(g_{1} y_{0}, \delta_{g_{1}}\right), \ldots, O\left(g_{k} y_{0}, \delta_{g_{k}}\right)\right\} .
$$

Let $\delta=\min \left\{\delta_{g_{1}}, \ldots, \delta_{g_{k}}\right\}$. We are going to check that then $d_{H}\left(F(y), F\left(y_{0}\right)\right)<\varepsilon$ whenever $y \in V$ and $\rho\left(y, y_{0}\right)<\delta$. 
Indeed, for every $g \in G, g y_{0}$ belongs to an element of the cover

$$
\left\{O\left(g_{1} y_{0}, \delta_{g_{1}}\right), \ldots, O\left(g_{k} y_{0}, \delta_{g_{k}}\right)\right\} \text {. }
$$

Without loss of generality, one can assume that $g y_{0} \in O\left(g_{1} y_{0}, \delta_{g_{1}}\right)$. Then for every $y \in O\left(y_{0}, \delta\right)$ we have

$$
\rho\left(g y, g_{1} y_{0}\right) \leq \rho\left(g y, g y_{0}\right)+\rho\left(g y_{0}, g_{1} y_{0}\right)=\rho\left(y, y_{0}\right)+\rho\left(g y_{0}, g_{1} y_{0}\right) \leq \delta+\delta_{g_{1}} \leq 2 \delta_{g_{1}} \text {. }
$$

Thus, $g y_{0}, g y \in O\left(g_{1} y_{0}, 2 \delta_{g_{1}}\right)$, which implies that $d_{H}\left(\varphi(g y), \varphi\left(g y_{0}\right)\right)<\varepsilon$. By the invariance of $d_{H}$, this yields

$$
d_{H}\left(g^{-1} \varphi(g y), g^{-1} \varphi\left(g y_{0}\right)\right)=d_{H}\left(\varphi(g y), \varphi\left(g y_{0}\right)\right)<\varepsilon \text { for all } g \in G,
$$

which in turn implies that

$$
d_{H}\left(\bigcup_{g \in G} g^{-1} \varphi(g y), \bigcup_{g \in G} g^{-1} \varphi\left(g y_{0}\right)\right)<\varepsilon .
$$

Thus, $d_{H}\left(F(y), F\left(y_{0}\right)\right)<\varepsilon$ for all $y \in O\left(y_{0}, \delta\right)$, proving the continuity of $F$ at the point $y_{0}$.

Remark 3.2. Recall that a hyperspace $\mathcal{E} \subset \exp X$ that satisfies the following condition is called an inclusion hyperspace: if $B \in \exp X$ and $A \in \mathcal{E}$ is such that $A \subset B$, then $B \in \mathcal{E}$. As is clear from the proof, Proposition 3.1 remains true also for any invariant inclusion hyperspace $\mathcal{E}$ instead of $\exp X$.

$(2) \Longrightarrow(3)$ follows directly from Theorem 2.7 .

Since every ANR (resp., AR) is locally continuum-connected (resp., connected and locally continuum-connected), the implication $(3) \Longrightarrow(1)$ follows from the following result.

Proposition 3.3. Let $G$ be a compact group and $X$ a metric $G$-space. Then:

(1) $X$ is connected iff $(\exp X) / G$ is connected; and

(2) if in addition $G$ is a Lie group, then $X$ is locally continuum-connected iff $(\exp X) / G$ is locally path-connected.

For the proof we need the following three lemmas.

Lemma 3.4. Let $G$ be a compact group, and $X$ a $G$-space containing a connected invariant subset (e.g., $G$ connected or $X[G] \neq \emptyset$ ). Then $X$ is connected iff $X / G$ is connected.

Proof. Only the "if" part requires a proof. Let $C \subset X$ be an invariant connected set. Assume the contrary, that $X / G$ is connected and $X$ is not connected. Then $X=A_{1} \cup A_{2}$ where the $A_{i}, i=1,2$, are nonempty, disjoint, closed-open subsets of $X$. Since $C$ is connected, one (and only one) of the sets $A_{i}, i=1,2$, contains $C$. Suppose $C \subset A_{1}$. By compactness of $G$, the orbit map $p: X \rightarrow X / G$ is open and closed. Hence the set $p\left(A_{2}\right)$ is a nonempty open-closed subset of $X / G$. Besides, by the invariance of $C$ the set $p\left(A_{2}\right)$ is disjoint from $p(C)$; so $p\left(A_{2}\right) \neq X / G$, which contradicts the connectedness of $X / G$.

Lemma 3.5. Let $G$ be a compact Lie group, and $X$ a $G$-space containing a pathconnected invariant subset (e.g., $G$ connected or $X[G] \neq \emptyset$ ). Then $X$ is pathconnected iff $X / G$ is path-connected. 
Proof. Only the "if" part requires a proof. Let $C \subset X$ be an invariant pathconnected set. It suffices to show that each point $x \in X$ can be joined with a point of $C$. Let $c \in C$ and let $l:[0,1] \rightarrow X / G$ be a path with $l(0)=p(x), l(1)=p(c)$, where $p: X \rightarrow X / G$ is the orbit projection. By [11, Ch. II, Theorem 6.2] there is a lifting $l^{\prime}:[0,1] \rightarrow X, p l^{\prime}=l$. Since $l^{\prime}(0)$ belongs to the orbit of $x$, there is $g \in G$ such that $x=g l^{\prime}(0)$. Then the path $g l^{\prime}:[0,1] \rightarrow X,\left(g l^{\prime}\right)(t)=g l^{\prime}(t)$, connects $x$ and $g l^{\prime}(1)$. But $g l^{\prime}(1) \in C$, because $l^{\prime}(1) \in C$ and $C$ is invariant. This completes the proof.

Lemma 3.6. Let $G$ be a compact Lie group. Then a $G$-space $X$ is locally pathconnected iff $X / G$ is locally path-connected.

Proof. Only the "if" part requires a proof. Let $x \in X$ and let $U$ be a neighborhood of $x$. Since the action of $G$ is continuous and $G$ is locally path-connected, one can choose a path-connected neighborhood $O$ of the unity in $G$ and a neighborhood $V$ of $x$ such that $O V=\{g v \mid g \in O, v \in V\} \subset U$. Without loss of generality, one can assume that $V$ is $G_{x}$-invariant. Since $G$ is a compact Lie group, there is a $G_{x}$-slice $R$ containing the point $x$ [26, Corollary 1.7.17]. Then the set $T=R \cap V$ is also a $G_{x}$-slice containing $x$. Since $X / G$ is locally path-connected, the neighborhood $p(G(T))$ of the point $p(x)$ contains a path-connected neighborhood $\widetilde{Y}$ of $p(x)$ in $X / G$. Let $Y=p^{-1}(\tilde{Y})$. Then $Y$ is a $G$-invariant neighborhood of the orbit $G(x)$ in $X$. Clearly, the set $S=T \cap Y$ contains $x$ and is a global $G_{x}$-slice for the $G$ space $Y$. Therefore the orbit spaces $S / G_{x}$ and $Y / G=\widetilde{Y}$ are homeomorphic [26] Proposition 1.7.6]; in particular, $S / G_{x}$ is path-connected. By Lemma 3.5] now $S$ is path-connected as well. Consequently, $O S$ is path-connected. Since $O S$ is an open neighborhood of $x$ and $O S \subset O T \subset O V \subset U$, we are done.

Proof of Proposition [3.3. 1. It is well known that $X$ is connected iff $\exp X$ is so; this is proved, for instance, in [23, Proposition 5.3.10] for $X$ compact, but the same proof is valid for noncompact $X$ as well. Hence, it remains to show that the connectedness of $(\exp X) / G$ implies that of $\exp X$. If $(\exp X) / G$ is connected, then the invariant subset $\Gamma$ of $\exp X$ consisting of all invariant, compact subsets of $X$ is connected. Indeed, the continuous surjection $\exp X \rightarrow \exp (X / G)$ induced by the orbit map $X \rightarrow X / G$ is invariant, and hence, it induces a continuous surjection $(\exp X) / G \rightarrow \exp (X / G)$. Therefore, if $(\exp X) / G$ is connected, then $\exp (X / G)$ is so, and since $\Gamma$ is homeomorphic to $\exp (X / G)$, it is connected too. Thus, $\Gamma$ is an invariant, connected subset of the $G$-space $\exp X$, and the connectedness of $(\exp X) / G$ implies, by Lemma 3.4, the connectedness of $\exp X$.

2. Respectively, $X$ is locally continuum-connected iff $\exp X$ is locally pathconnected (see [13, Lemma 1.4] and the final part of the proof of 13, Theorem 1.6]). By Lemma 3.6, $\exp X$ is locally path-connected iff $(\exp X) / G$ is locally pathconnected, and the proof is complete. Thus, Theorem 1.1 is completely proved.

Corollary 3.7. Let $G$ be a compact, metrizable, locally path-connected group, and $H \subset G$ a closed subgroup. Then $(\exp (G / H)) / G$ is an ANR. If in addition $G / H$ is connected, then $(\exp (G / H)) / G$ is an $A R$.

Proof. Under the hypothesis the coset space $G / H$ is locally path-connected, and hence, locally continuum-connected. Besides, since the quotient map $G \rightarrow G / H$ is perfect, $G / H$ is metrizable too (see, e.g., [15, Section XI, Theorem 5.2(3)]). Then 
by Proposition $3.3 \exp (G / H) \in G$-ANR; if in addition $G / H$ is connected, then $\exp (G / H) \in G$-AR. Now the result follows immediately from Theorem 2.7.

Since every compact Lie group $G$ is locally path-connected (moreover, it is a $G$ ANR [26. Corollary 1.6.7]), we get from Corollary 3.7 the following positive answer to the first question of West's Problem:

Corollary 3.8. Let $G$ be a compact Lie group. Then $(\exp G) / G$ is an ANR. If in addition $G$ is connected, then $(\exp G) / G$ is an $A R$.

In combination with [17, Corollary 2], Theorem 1.1 yields also the following fact.

Corollary 3.9. Let $G$ be a finite group acting on a nondegenerate Peano continuum $X$. Then the orbit space $(\exp X) / G$ is a Hilbert cube.

\section{The $G$-NERVE}

In this section we develop a necessary technique involving the notion of a $G$ nerve. The results proved here will be applied in the next section.

Following Matumoto 22, we define the $G$-nerve $\mathcal{N}(\mathcal{U})$ of a $G$-normal cover $\mathcal{U}$.

Let $G$ be a compact Lie group and $\mathcal{H}=\left\{H_{\mu} \mid \mu \in M\right\}$ a family of closed subgroups of $G$. The Milnor join $\mathcal{J}$ of the family of cosets $\left\{G / H_{\mu} \mid \mu \in M\right\}$ is defined as follows. Let $\mathcal{J}^{\prime}$ be the following set:

$$
\left\{\left(t_{\mu}, g_{\mu} H_{\mu}\right)_{\mu \in M} \in \prod_{\mu \in M}\left(I \times G / H_{\mu}\right) \mid t_{\mu} \neq 0 \text { for only finite } \mu \text { 's and } \sum_{\mu \in M} t_{\mu}=1\right\} .
$$

We let

$$
\left(t_{\mu}, g_{\mu} H_{\mu}\right)_{\mu \in M} \sim\left(s_{\mu}, h_{\mu} H_{\mu}\right)_{\mu \in M}
$$

iff $t_{\mu}=s_{\mu}$ for all $\mu \in M$, and $g_{\mu} H_{\mu}=h_{\mu} H_{\mu}$ whenever $t_{\mu} \neq 0$. Then $\sim$ is an equivalence relation on $\mathcal{J}^{\prime}$, and we shall denote by $\mathcal{J}$ the quotient set $\mathcal{J}^{\prime} / \sim$.

In what follows we shall use the convention $\sum_{\mu \in M} t_{\mu} g_{\mu} H_{\mu}$ for the equivalence class of the point $\left(t_{\mu}, g_{\mu} H_{\mu}\right)_{\mu \in M}$. The numbers $t_{\mu}$ are called barycentric coordinates of the point $\sum_{\mu \in M} t_{\mu} g_{\mu} H_{\mu} \in \mathcal{J}$.

For any finite subset $\left\{\mu_{0}, \ldots, \mu_{n}\right\} \subset M$, we consider the following subset of $\mathcal{J}$ :

$$
G / H_{\mu_{0}} * \cdots * G / H_{\mu_{n}}=\left\{\sum_{\mu \in M} t_{\mu} g_{\mu} H_{\mu} \in \mathcal{J} \mid t_{\mu}=0 \text { for all } \mu \notin\left\{\mu_{0}, \ldots, \mu_{n}\right\}\right\} .
$$

Observe that each $G / H_{\mu_{0}} * \cdots * G / H_{\mu_{n}}$ with its quotient topology is a compact metrizable space, which is called the Milnor join of the spaces $G / H_{\mu_{0}}, \ldots, G / H_{\mu_{n}}$ (see [24]).

We topologize $\mathcal{J}$ by the weak topology with respect to the family of all finite subjoins, i.e., a set $U \subset \mathcal{J}$ is open in $\mathcal{J}$ whenever $U \cap\left(G / H_{\mu_{0}} * \cdots * G / H_{\mu_{n}}\right)$ is open in $G / H_{\mu_{0}} * \cdots * G / H_{\mu_{n}}$ for any finite subjoin $G / H_{\mu_{0}} * \cdots * G / H_{\mu_{n}} \subset \mathcal{J}$. It is easy to check that $\mathcal{J}$ becomes a $G$-space if we define the action of $G$ as follows:

$$
g\left(\sum_{\mu \in M} t_{\mu} g_{\mu} H_{\mu}\right)=\sum_{\mu \in M} t_{\mu} g g_{\mu} H_{\mu}, \quad g \in G .
$$


Next, if $g_{\mu_{0}} H_{\mu_{0}} \in G / H_{\mu_{0}}, \ldots, g_{\mu_{n}} H_{\mu_{n}} \in G / H_{\mu_{n}}$ are fixed elements, then we will denote by $\left\langle g_{\mu_{0}} H_{\mu_{0}}, \ldots, g_{\mu_{n}} H_{\mu_{n}}\right\rangle$ the subspace

$$
\begin{aligned}
& \left\{\sum_{\mu \in M} t_{\mu} g_{\mu}^{\prime} H_{\mu} \in \mathcal{J} \mid t_{\mu}=0 \text { for } \mu \notin\left\{\mu_{0}, \ldots, \mu_{n}\right\}\right. \\
& \left.\quad \text { and } g_{\mu_{i}}^{\prime} H_{\mu_{i}}=g_{\mu_{i}} H_{\mu_{i}} \text { for } 0 \leq i \leq n\right\}
\end{aligned}
$$

of $G / H_{\mu_{0}} * \cdots * G / H_{\mu_{n}}$, which is called an $n$-cell.

Let $G$ be a compact Lie group and $X$ a $G$-space. For each index $\mu \in M$, let $H_{\mu}$ be a closed subgroup of $G$, and let $S_{\mu}$ be an $H_{\mu}$-slice in $X$. Then the family

$$
\mathcal{U}=\left\{\left(g S_{\mu}, H_{\mu}\right) \mid g \in G, \mu \in M\right\}
$$

consisting of tubular slice-sets $g S_{\mu}$ with companion groups $H_{\mu}$ is called a $G$-normal cover of $X$ if the family of open tubes $\left\{G\left(S_{\mu}\right) \mid \mu \in M\right\}$ covers $X$ and there exists a locally finite invariant partition of unity $\left\{\varphi_{\mu}: X \rightarrow[0,1] \mid \mu \in M\right\}$ subordinated to $\mathcal{U}$, i.e., each $\varphi_{\mu}$ is an invariant function with $\varphi_{\mu}^{-1}((0,1]) \subset G\left(S_{\mu}\right)$ and the supports $\left\{\varphi_{\mu}^{-1}((0,1]) \mid \mu \in M\right\}$ constitute a locally finite family.

Let $\widetilde{\mathcal{N}}(\mathcal{U})$ be the ordinary nerve of the invariant cover $\left\{G\left(S_{\mu}\right) \mid \mu \in M\right\}$. In the sequel we will denote by $\left\langle\mu_{0}, \ldots, \mu_{n}\right\rangle$ the simplex of $\tilde{\mathcal{N}}(\mathcal{U})$ constituted by the sets $G\left(S_{\mu_{0}}\right), \ldots, G\left(S_{\mu_{n}}\right)$. Let $f_{\mu}: G\left(S_{\mu}\right) \rightarrow G / H_{\mu}$ be the corresponding $G$-map with $f_{\mu}^{-1}\left(e H_{\mu}\right)=S_{\mu}$ (see Slice Theorem 2.2). For any simplex $L=\left\langle\mu_{0}, \ldots, \mu_{n}\right\rangle \in \widetilde{\mathcal{N}}(\mathcal{U})$, we define the following subset of the product $\prod_{i=0}^{n} G / H_{\mu_{i}}$ :

$$
F_{L}=\left\{\left(f_{\mu_{0}}(x), \ldots, f_{\mu_{n}}(x)\right) \mid x \in \bigcap_{i=0}^{n} G\left(S_{\mu_{i}}\right)\right\} .
$$

It follows from the equivariance of $f_{\mu_{i}}$ that $F_{L}$ is an invariant subset of the $G$-space $\prod_{i=0}^{n} G / H_{\mu_{i}}$.

Denote by $\mathcal{F}$ the family of all these sets $F_{L}$. Let $\Delta\left(L, F_{L}\right)$ be the subset of the finite subjoin $G / H_{\mu_{0}} * \cdots * G / H_{\mu_{n}}$ of $\mathcal{J}$ consisting of all those points $\sum_{i=0}^{n} t_{i} g_{i} H_{\mu_{i}}$ for which $\left(g_{0} H_{\mu_{0}}, \ldots, g_{n} H_{\mu_{n}}\right) \in F_{L}$ and $\left(t_{0}, \ldots, t_{n}\right) \in \Delta^{n}$. Clearly, $\Delta\left(L, F_{L}\right)$ is invariant in $G / H_{\mu_{0}} * \cdots * G / H_{\mu_{n}}$, and hence, in $\mathcal{J}$.

We call $\Delta\left(L, F_{L}\right)$ a $G$-n-simplex over the simplex $L$ along the set $F_{L}$. The homogeneous $G$-spaces $G / H_{\mu_{0}}, \ldots, G / H_{\mu_{n}}$ are called $G$-vertices of $\Delta\left(L, F_{L}\right)$.

The $G$-nerve of the cover $\mathcal{U}$ is, by definition, the union

$$
\mathcal{N}(\mathcal{U})=\bigcup\left\{\Delta\left(L, F_{L}\right) \mid L \in \tilde{\mathcal{N}}(\mathcal{U}), F_{L} \in \mathcal{F}\right\}
$$

equipped with the topology induced from $\mathcal{J}$. It is not difficult to check that the topology of $\mathcal{N}(\mathcal{U})$ is the weak one with respect to its closed, invariant cover $\left\{\Delta\left(L, F_{L}\right) \mid L \in \tilde{\mathcal{N}}(\mathcal{U}), F_{L} \in \mathcal{F}\right\}$. That is to say, a subset $W \subset \mathcal{N}(\mathcal{U})$ is open iff $W \cap \Delta\left(L, F_{L}\right)$ is open in $\Delta\left(L, F_{L}\right)$ for each $G$-simplex $\Delta\left(L, F_{L}\right)$ in $\mathcal{N}(\mathcal{U})$. Since $\mathcal{N}(\mathcal{U})$ is an invariant subset of $\mathcal{J}$, it becomes a $G$-space with respect to the action induced from $\mathcal{J}$.

The $G$ - $n$-skeleton $\mathcal{N}(\mathcal{U})^{n}$ of $\mathcal{N}(\mathcal{U})$ is defined to be the union of all $G$ - $k$-simplexes in $\mathcal{N}(\mathcal{U})$ with $k \leq n$. 
If $g H_{\lambda}$ is a vertex of the $G$-nerve $\mathcal{N}(\mathcal{U})$, then its star $S t\left(g H_{\lambda}, \mathcal{N}(\mathcal{U})\right.$ is defined to be the union of all cells for which $g H_{\lambda}$ is a vertex. The $G$-carrier of a point $x \in \mathcal{N}(\mathcal{U})$ is defined to be the smallest $G$-simplex of $\mathcal{N}(\mathcal{U})$ containing $x$. The cell in the $G$-carrier that contains $x$ is called the carrier of $x$.

Recall that a cover $\mathcal{U}$ of a space $X$ is called a star-refinement of a cover $\mathcal{V}$ whenever for every $U \in \mathcal{U}$ there exists an element $V \in \mathcal{V}$ that contains the star $S t(U, \mathcal{U})$ of $U$ with respect of $\mathcal{U}$; here $S t(U, \mathcal{U})=\{W \in \mathcal{U} \mid W \cap U \neq \emptyset\}$.

Lemma 4.1. Let $X$ be a paracompact $G$-space. Then for each open cover $\mathcal{V}$ of $X$ there exists a $G$-normal cover $\mathcal{U}=\left\{\left(g S_{\lambda}, H_{\lambda}\right) \mid g \in G, \lambda \in \Lambda\right\}$ of $X$ such that $\mathcal{U}$ is a star-refinement of $\mathcal{V}$.

Proof. Since $X$ is paracompact, one can choose open covers $\mathcal{U}_{1}$ and $\mathcal{U}_{2}$ of $X$ such that $\mathcal{U}_{1}$ is a star-refinement of $\mathcal{U}_{2}$ and $\mathcal{U}_{2}$ is a star-refinement of $\mathcal{V}$.

Let us denote by $U$ the subset of $X \times X$ consisting of all those pairs $(x, y)$ such that there exists an element $O \in U_{1}$ that contains both $x$ and $y$. Clearly $U$ is an open neighborhood of the diagonal $\Delta \subset X \times X$. By compactness of $G$, there is an invariant neighborhood $V$ of $\Delta$ in $X \times X$ such that $V \subset U$. Define

$$
\mathcal{W}=\{V[x] \mid x \in X\}, \quad \text { where } V[x]=\{z \in X \mid(x, z) \in V\} .
$$

Then $\mathcal{W}$ is an open $G$-cover of $X$, and $V[x] \subset U[x]=S t\left(x, \mathcal{U}_{1}\right)$ for each $x \in X$. Since $\operatorname{St}\left(x, \mathcal{U}_{1}\right)$ is contained in an element of $\mathcal{U}_{2}$, we infer that $\mathcal{W}$ is a refinement of $\mathcal{U}_{2}$, and hence, a star-refinement of $\mathcal{U}$.

Next, we fix on each orbit $G(x) \subset X$ a point, say $x \in G(x)$, and choose an element $W_{x} \in \mathcal{W}$ such that $x \in W_{x}$. By the continuity of the action of $G$ on $X$ there exist a neighborhood $O_{x}$ of the unity in $G$ and a $G_{x}$-invariant neighborhood $N_{x}$ of $x$ in $X$ such that $O_{x} N_{x} \subset W_{x}$. By Slice Theorem 2.2, there exists a $G_{x^{-}}$ slice $Q_{x}$ such that $x \in Q_{x}$. Then the set $S_{x}=Q_{x} \cap N_{x}$ is also a $G_{x}$-slice, and $x \in S_{x} \subset N_{x}$. Hence $O_{x} S_{x} \subset W_{x}$. We define $\mathcal{U}$ to be the totality of all these slicesets $\left(g S_{x}, G_{x}\right), g \in G, G(x) \in X / G$. Since the orbit map $X \rightarrow X / G$ is closed, we see that $X / G$ is paracompact too [15, Section VIII, Theorem 2.4]. This implies that the invariant cover $\left\{G\left(S_{x}\right)\right\}$ admits a locally finite partition of unity subordinated to $\mathcal{U}$, and hence, $\mathcal{U}$ is an open $G$-normal cover. Since $g S_{x} \subset g W_{x}$ and $g W_{x} \in \mathcal{W}$, we conclude that $\mathcal{U}$ is a refinement of $\mathcal{W}$, and since $\mathcal{W}$ is a star-refinement of $\mathcal{V}$, we infer that $\mathcal{U}$ is a star-refinement of $\mathcal{V}$.

Lemma 4.2. Let $Y$ be a $G$-space, and let $\mathcal{U}=\left\{\left(g S_{\mu}, H_{\mu}\right) \mid g \in G, \mu \in M\right\}$ be a $G$-normal cover of $Y$. Then for each locally finite invariant partition of unity subordinated to $\mathcal{U}$, there exists a $G$-map $p: Y \rightarrow \mathcal{N}(\mathcal{U})$ such that $p^{-1}\left(\operatorname{St}\left(g H_{\mu}, \mathcal{N}(\mathcal{U})\right)\right) \subset$ $G\left(S_{\mu}\right)$ for any $g \in G$ and $\mu \in M$, where

$$
S t\left(g H_{\mu}, \mathcal{N}(\mathcal{U})\right)=\left\{\sum_{\lambda \in M} t_{\lambda} g_{\lambda} H_{\lambda} \in \mathcal{N}(\mathcal{U}) \mid t_{\mu}>0, g_{\mu}=g\right\} .
$$

Proof. Let $\left\{\varphi_{\mu}: Y \rightarrow[0,1] \mid \mu \in M\right\}$ be a locally finite invariant partition of unity subordinated to $\mathcal{U}$, i.e., $\varphi_{\mu}^{-1}((0,1]) \subset G\left(S_{\mu}\right)$ for all $\mu \in M$. Then we define the canonical $G$-map $p: Y \rightarrow \mathcal{N}(\mathcal{U})$ as follows.

Since $\left\{\varphi_{\mu}: Y \rightarrow[0,1] \mid \mu \in M\right\}$ is locally finite, for each $y \in Y$ there are only a finite number of indices, say $\mu_{0}, \ldots, \mu_{n}$, such that $\varphi_{\mu_{i}}(y) \neq 0, i=0, \ldots, n$. Let $\left\langle f_{\mu_{0}}(y), \ldots, f_{\mu_{n}}(y)\right\rangle$ be the corresponding $n$-cell in $G / H_{\mu_{0}} * \cdots * G / H_{\mu_{n}}$. Then by 
definition, $p(y)$ is the point of $\left\langle f_{\mu_{0}}(y), \ldots, f_{\mu_{n}}(y)\right\rangle$ with the barycentric coordinates $\varphi_{\mu_{0}}(y), \ldots, \varphi_{\mu_{n}}(y)$, i.e.,

$$
p(y)=\sum_{i=0}^{n} \varphi_{\mu_{i}}(y) f_{\mu_{i}}(y) .
$$

We claim that $p$ is continuous. For, let $y_{0} \in Y$ be an arbitrary point. Using the local finiteness of the partition of unity $\left\{\varphi_{\mu} \mid \mu \in M\right\}$, we take a neighborhood $V$ of $y_{0}$ in $Y$ such that only for a finite number of indices $\mu_{0}, \ldots, \mu_{m}$ is $\varphi_{\mu_{i}}(y) \neq 0$ for $y \in V$. Then

$$
p(y)=\sum_{i=0}^{m} \varphi_{\mu_{i}}(y) f_{\mu_{i}}(y) \text { for all } y \in V .
$$

Now the continuity of $p$ in $V$ follows from the continuity of the maps $f_{\mu_{i}}$ and $\varphi_{\mu_{i}}$, $i=0, \ldots, m$, in $V$.

For a space $X$ we will denote by $\mathcal{F}_{n}(X)$ the subset of $\exp X$ that consists of all those sets $A \subset X$ that have at most $n$ elements. By $\mathcal{F}_{\infty}(X)$ we shall denote the union $\bigcup_{n=1}^{\infty} \mathcal{F}_{n}(X)$.

Lemma 4.3. Let $P$ be a polyhedron and $P^{1}$ its 1-dimensional skeleton. Then there is a continuous map $\xi: P \rightarrow \mathcal{F}_{\infty}\left(P^{1}\right)$ such that

(1) $\xi(z)=\{z\}$ for all $z \in P^{1}$, and

(2) if $\tau$ is the carrier of $x \in P$ and $\operatorname{dim} \tau=n$, then $\xi(x)$ is contained in the 1 -skeleton of $\tau$ and contains at most $3^{n-1}$ points.

Proof. Let $P^{n}, n \geq 1$, denote the $n$-skeleton of $P$, and let $\xi_{1}: P^{1} \rightarrow P^{1}$ be the identity map. From the proof of [23, Proposition 8.4.2], we get the following fact. Claim. For every $n \geq 1$, there is a continuous map $\xi_{n}: P^{n} \rightarrow \mathcal{F}_{\infty}\left(P^{1}\right)$ such that

a. if $\tau$ is the carrier of $x \in P$ and $\operatorname{dim} \tau=n$, then $\xi_{n}(x)$ is contained in the 1-skeleton of $\tau$ and contains at most $3^{n-1}$ points, and

b. $\xi_{n+1}$ extends $\xi_{n}$ for all $n \geq 1$.

Now we define the required map $\xi$ to be equal to $\xi_{n}$ on the $n$-dimensional skeleton $P^{n}$.

In the next lemma, for a given simplex $L=\left\langle\mu_{0}, \ldots, \mu_{n}\right\rangle \subset \widetilde{\mathcal{N}}(\mathcal{U})$, a given $n$-cell $\left\langle g_{\mu_{0}} H_{\mu_{0}}, \ldots, g_{\mu_{n}} H_{\mu_{n}}\right\rangle \subset \mathcal{N}(\mathcal{U})$ and the corresponding $G$-n-simplex $\sigma=\Delta\left(L, F_{L}\right)$, we shall use the following notation:

$$
\partial\left\langle g_{\mu_{0}} H_{\mu_{0}}, \ldots, g_{\mu_{n}} H_{\mu_{n}}\right\rangle=\left\{\sum_{i=0}^{n} t_{i} g_{\mu_{i}} H_{\mu_{i}} \mid\left(t_{0}, \ldots, t_{n}\right) \in \partial \Delta^{n}\right\},
$$

where we use the same notation $\partial \Delta^{n}$ for the ordinary boundary of the standard simplex $\Delta^{n}$. Correspondingly,

$$
\partial \sigma=\left\{\sum_{i=0}^{n} t_{i} g_{\mu_{i}} H_{\mu_{i}} \mid\left(t_{0}, \ldots, t_{n}\right) \in \partial \Delta^{n}, \quad\left(g_{\mu_{0}} H_{\mu_{0}}, \ldots, g_{\mu_{n}} H_{\mu_{n}}\right) \in F_{L}\right\} .
$$

In what follows we shall need the following equivariant version of Lemma 4.3:

Lemma 4.4. Let $\mathcal{N}(\mathcal{U})$ be a $G$-nerve and $\Gamma$ its $G$-1-dimensional skeleton. Then there is a $G$-map $R: \mathcal{N}(\mathcal{U}) \rightarrow \mathcal{F}_{\infty}(\Gamma)$ such that

(1) $R(z)=\{z\}$ for all $z \in \Gamma$, and 
(2) if $s$ is the carrier of $x \in \mathcal{N}(\mathcal{U})$, then $R(x)$ is contained in the 1-skeleton of s. More precisely, if $\operatorname{dim} s=n$, then $R(x) \in \mathcal{F}_{3^{n-1}}(s)$.

Proof. We are going to apply Lemma 4.3 above. In our case $P$ is the polyhedron accompanying the $G$-nerve $\mathcal{N}(\mathcal{U})$, i.e., the polyhedron $\tilde{\mathcal{N}}(\mathcal{U})$. Let $K$ be its 1skeleton and $\xi: P \rightarrow \mathcal{F}_{\infty}(K)$ the continuous map from Lemma 4.3.

Let $x \in \mathcal{N}(\mathcal{U})$ and let the $G$-simplex

$$
\sigma=\Delta\left(L, F_{L}\right) \subset G / H_{\mu_{0}} * \cdots * G / H_{\mu_{n}}
$$

be the $G$-carrier of $x$. Then $x=\sum_{i=0}^{n} t_{i} g_{\mu_{i}} H_{\mu_{i}}$, where $s=\left\langle g_{\mu_{0}} H_{\mu_{0}}, \ldots, g_{\mu_{n}} H_{\mu_{n}}\right\rangle$ is the carrier of $x$.

Define the map $R: \mathcal{N}(\mathcal{U}) \rightarrow \mathcal{F}_{\infty}(\Gamma)$ by setting

$$
\begin{aligned}
R(x)= & R\left(\sum_{i=0}^{n} t_{i} g_{\mu_{i}} H_{\mu_{i}}\right)=\xi\left(t_{0}, \ldots, t_{n}\right) \cdot\left(g_{\mu_{0}} H_{\mu_{0}}, \ldots, g_{\mu_{n}} H_{\mu_{n}}\right) \\
& =\left\{\sum_{i=0}^{n} u_{i} g_{\mu_{i}} H_{\mu_{i}} \mid\left(u_{0}, \ldots, u_{n}\right) \in \xi\left(t_{0}, \ldots, t_{n}\right)\right\} .
\end{aligned}
$$

Since $\xi\left(t_{0}, \ldots, t_{n}\right)$ belongs to $\mathcal{F}_{3^{n-1}}\left(P^{1}\right)$, we see that $R(x)$ belongs to $\mathcal{F}_{3^{n-1}}(s) \subset$ $\mathcal{F}_{3^{n-1}}(\Gamma)$. Continuity and equivariance of $R$ are evident from the definition of $R$. Properties (1) and (2) follow from the analogous properties in Lemma 4.3

\section{Proofs of Theorems 1.3 and 1.4}

We shall give a sequence of lemmas culminating in proofs of Theorems 1.3 and 1.4 .

In this section $d$ will always denote the Euclidean metric on $\mathbb{R}^{n}$.

By $\mathcal{P}(n)$ we will denote the subset of $L(n)$ consisting of all compact convex bodies $A$ such that the contact set $\partial A \cap \partial B^{n}$ has an empty interior in the boundary sphere $S^{n-1}=\partial B^{n}$.

Lemma 5.1. For each $\varepsilon>0$ and each body $E \in L_{0}(n)$ there exists a body $D \in \mathcal{P}(n)$ such that $d_{H}(E, D)<\varepsilon$ and the $O(n)$-stabilizer $O(n)_{E}$ of $E$ coincides with the $O(n)$-stabilizer $O(n)_{D}$ of $D$.

Proof. Let $r: \mathcal{B}(n) \rightarrow L(n)$ be the $O(n)$-equivariant retraction from Theorem 2.3.

Because of compactness of $L(n)$ (Theorem [2.4), one can find a real $0<\delta<\varepsilon / 2$ such that $d_{H}(r(A), A)<\varepsilon / 2$ for all $A$ belonging to the $\delta$-neighborhood of $L(n)$ in $\mathcal{B}(n)$, where $d_{H}$ denotes the Hausdorff metric on $\mathcal{B}(n)$.

Let $K$ be the stabilizer $O(n)_{E}$. It follows from Slice Theorem 2.2 that there is a real $0<\eta<\delta$ such that the inequality $d_{H}(E, X)<\eta$ for $X \in L_{0}(n)$ implies that the stabilizer $O(n)_{X}$ is conjugate to a subgroup of $K$, i.e., $\left(O(n)_{X}\right) \preceq(K)$.

Choose a centrally symmetric, convex polyhedron $P \subset \mathbb{R}^{n}$ with a nonempty interior, such that $d_{H}(E, P)<\eta, P \subset E$ and all the vertices $p_{1}, \ldots, p_{k}$ of $P$ lie on the boundary $\partial E$. Then the convex hull

$$
M=\operatorname{conv}\left(K\left(p_{1}\right) \cup \cdots \cup K\left(p_{k}\right)\right)
$$

is a centrally symmetric, compact, convex, $K$-invariant subset of $\mathbb{R}^{n}$. Since it contains $P$, we see that $M$ has a nonempty interior in $\mathbb{R}^{n}$, and so $M \in \mathcal{B}(n)$.

We claim that the boundary $\partial M$ does not contain an $(n-1)$-dimensional elliptic domain, i.e., an open subset $V \subset \partial M$ which is at the same time an open subset 
of some $(n-1)$-dimensional ellipsoid surface lying in $\mathbb{R}^{n}$. It suffices to show that none of the orbits $K\left(p_{i}\right), i=1, \ldots, k$, contains an $(n-1)$-dimensional elliptic domain. Assume the contrary, that some $K\left(p_{i}\right)$ contains an $(n-1)$-dimensional elliptic domain $V$. Since $K\left(p_{i}\right)$ lies on the $(n-1)$-dimensional sphere $\partial B\left(0,\left\|p_{i}\right\|\right)$ centered at the origin and having the radius $\left\|p_{i}\right\|$, then $V$ should be, in fact, a domain of the sphere $\partial B\left(0,\left\|p_{i}\right\|\right)$. Since $K\left(p_{i}\right)$ is homogeneous and compact, we conclude that there are finitely many open subsets $V_{1}, \ldots, V_{n}$ of $K\left(p_{i}\right)$ such that $K\left(p_{i}\right)=V_{1} \cup \cdots \cup V_{n}$, where each $V_{j}$ is homeomorphic to $V$. Next, by the Domain Invariance Theorem (see, e.g., [27 Ch. 4, Section 7, Theorem 16]), each $V_{j}$ should be open in the sphere $\partial B\left(0,\left\|p_{i}\right\|\right)$, and hence, the union $V_{1} \cup \cdots \cup V_{n}$ is open in $\partial B\left(0,\left\|p_{i}\right\|\right)$. But $V_{1} \cup \cdots \cup V_{n}$ is also closed in $\partial B\left(0,\left\|p_{i}\right\|\right)$, because it is equal to $K\left(p_{i}\right)$. Now, by connectedness of $\partial B\left(0,\left\|p_{i}\right\|\right)$, it then follows that $K\left(p_{i}\right)$ must be the whole sphere $\partial B\left(0,\left\|p_{i}\right\|\right)$. Consequently, $K$ acts transitively on the sphere $\partial B\left(0,\left\|p_{i}\right\|\right)$, and hence on the unit sphere $S^{n-1}$. This contradiction proves the claim.

In particular, the contact set of $M$, which is by definition the intersection of $\partial M$ with the boundary of the Löwner ellipsoid $l(M)$, also does not contain an elliptic domain.

Now consider the body $D=r(M) \in L(n)$. Since $D=T(M)$ for some linear nondegenerate operator $T: \mathbb{R}^{n} \rightarrow \mathbb{R}^{n}$, the contact set $\partial D \cap \partial B^{n}$ is just the image under $T$ of the contact set $\partial M \cap \partial(l(M))$. Consequently, $\partial D \cap \partial B^{n}$ has an empty interior in the sphere $S^{n-1}=\partial B^{n}$, and hence, $D \in \mathcal{P}(n)$. Since $d_{H}(P, E)<\eta$ and $P \subset M \subset E$, we see that $d_{H}(M, E)<\eta<\delta$. Consequently, $d_{H}(D, E)<\varepsilon$, which implies in turn that $\left(O(n)_{D}\right) \preceq(K)$. Since $M$ is $K$-invariant, we have $K \subset O(n)_{M}$, and since $r$ is $O(n)$-equivariant, $O(n)_{M} \subset O(n)_{r(M)}=O(n)_{D}$. Thus $K \subset O(n)_{D}$, which implies in combination with $\left(O(n)_{D}\right) \preceq(K)$ that $O(n)_{D}=K$.

The following lemma is the key in the proof of Theorems 1.3 and 1.4

Lemma 5.2 (Key lemma). Let $\varepsilon>0$, and let $\mathcal{V}_{\varepsilon}$ be the $\varepsilon$-cover of $L_{0}(n)$. Then there exist a $G$-normal cover $\mathcal{U}=\left\{\left(g S_{\lambda}, H_{\lambda}\right) \mid g \in G, \lambda \in \Lambda\right\}$ of $L_{0}(n)$ and $G$-maps $p: L_{0}(n) \rightarrow \mathcal{N}(\mathcal{U}), \quad \Phi: \mathcal{N}(\mathcal{U}) \rightarrow \mathcal{P}(n)$ such that

(1) for each $g S_{\lambda} \in \mathcal{U}$ there is an element $V(g, \lambda) \in \mathcal{V}_{\varepsilon / 2}$ with $g S_{\lambda} \subset V(g, \lambda)$ and $\Phi\left(S t\left(g H_{\lambda}, \mathcal{N}(\mathcal{U})\right)\right) \subset V(g, \lambda)$, and

(2) the composition $\Phi p$ is $\varepsilon$-close to the identity map of $L_{0}(n)$.

Proof. By Lemma 4.1 there is a $G$-normal cover

$$
\mathcal{U}=\left\{\left(g S_{\lambda}, H_{\lambda}\right) \mid g \in G, \lambda \in \Lambda\right\}
$$

of $L_{0}(n)$ that is a star-refinement of $\mathcal{V}_{\varepsilon / 4}$. Fix an invariant locally finite partition of unity $\left\{\varphi_{\lambda}\right\}_{\lambda \in \Lambda}$ subordinated to $\mathcal{U}$. Let $p: L_{0}(n) \rightarrow \mathcal{N}(\mathcal{U})$ be the $G$-map corresponding to $\left\{\varphi_{\lambda}\right\}_{\lambda \in \Lambda}$ (see Lemma 4.2).

For every $g S_{\lambda} \in \mathcal{U}$ we choose an element $V(g, \lambda) \in \mathcal{V}_{\varepsilon / 2}$ such that

$$
\operatorname{St}\left(g S_{\lambda}, \mathcal{U}\right) \subset V(g, \lambda) / 2,
$$

where $V(g, \lambda) / 2 \in \mathcal{V}_{\varepsilon / 4}$ denotes the open ball in $L_{0}(n)$ concentric with $V(g, \lambda)$ and having half the radius of $V(g, \lambda)$.

Now we define the map $\Phi: \mathcal{N}(\mathcal{U}) \rightarrow \mathcal{P}(n)$ as follows.

First we define a $G$-map $q: \Gamma \rightarrow \mathcal{P}(n)$, where $\Gamma$ is the $G$-1-skeleton of $\mathcal{N}(\mathcal{U})$. 
For each $G$-vertex $G / H_{\lambda} \in \mathcal{N}(\mathcal{U})$ with $H_{\lambda}$ the subgroup corresponding to the $H_{\lambda}$-slice $S_{\lambda} \in \mathcal{U}$, we select a point $X_{\lambda} \in S_{\lambda}$ such that the stabilizer $G_{X_{\lambda}}$ coincides with the group $H_{\lambda}$.

By Lemma 5.1 above, we choose a body $A_{\lambda} \in \mathcal{P}(n), \varepsilon / 4$-close to $X_{\lambda}$ and having the stabilizer $G_{A_{\lambda}}=H_{\lambda}$.

Define the $G$-map $q: G / H_{\lambda} \rightarrow G\left(A_{\lambda}\right) \subset \mathcal{P}(n)$ by setting

$$
q\left(g H_{\lambda}\right)=g A_{\lambda} \text { for every } g H_{\lambda} \in G / H_{\lambda} .
$$

The inclusion $H_{\lambda} \subset G_{A_{\lambda}}$ guarantees that $q$ is a well-defined $G$-map. We aim to extend $q$ equivariantly to the $G$-1-skeleton $\Gamma$ of $\mathcal{N}(\mathcal{U})$. It suffices to extend $q$ over each $G$-1-simplex of $\Gamma$. Let $\Delta$ be a $G$-1-simplex of $\Gamma$ with the $G$-vertices $G / H_{\lambda}$ and $G / H_{\mu}$. We first define two $G$-maps $s_{1}: \Delta \rightarrow \mathcal{B}(n)$ and $s_{2}: \Delta \rightarrow \mathcal{B}(n)$. Indeed, let $y=t g_{0} H_{\lambda}+(1-t) g_{1} H_{\mu}$ be an arbitrary point of $\Delta$, where $g_{0}, g_{1} \in G$ and $g_{0} S_{\lambda} \cap g_{1} S_{\mu} \neq \emptyset$.

We set

$$
s_{1}(y)=t g_{0} A_{\lambda}+(1-t) g_{1} A_{\mu} \quad \text { and } \quad s_{2}(y)=g_{0} A_{\lambda} .
$$

Recall that here $t W+(1-t) Z$ means the Minkowski convex combination of the convex sets $W, Z \subset \mathbb{R}^{n}$, i.e.,

$$
t W+(1-t) Z=\{t w+(1-t) z \mid w \in W, z \in Z\} .
$$

Clearly $s_{1}$ and $s_{2}$ are continuous $G$-maps from $\Delta$ to $\mathcal{B}(n)$.

Define $q^{\prime}(y)$ to be the convex hull of the union $s_{1}(y) \cup s_{2}(y)$.

Since $s_{1}(y) \cup s_{2}(y)$ depends continuously upon $y \in \Delta$, the continuity of $q^{\prime}$ now follows from the continuity of the convex hull operator (see [31, Theorem 2.7.4(iv)]).

Analogously, let $q^{\prime \prime}(y)$ be the convex hull of the union

$$
g_{1} A_{\mu} \cup\left(t g_{0} A_{\lambda}+(1-t) g_{1} A_{\mu}\right) .
$$

For the same reason, $q^{\prime \prime}(y)$ depends continuously upon $y \in \Delta$.

Now we paste $q^{\prime}$ and $q^{\prime \prime}$ to define the desired map $q$ :

$$
q\left(t g_{0} H_{\lambda}+(1-t) g_{1} H_{\mu}\right)=\left\{\begin{array}{cl}
q^{\prime}\left((1-2 t) g_{0} H_{\lambda}+2 t g_{1} H_{\mu}\right), & \text { if } 0 \leq t \leq 1 / 2, \\
q^{\prime \prime}\left((2 t-1) g_{1} H_{\mu}+(2-2 t) g_{0} H_{\lambda}\right), & \text { if } 1 / 2 \leq t \leq 1 .
\end{array}\right.
$$

Since $(1-2 t) g_{0} H_{\lambda}+2 t g_{1} H_{\mu}$ and $(2 t-1) g_{1} H_{\mu}+(2-2 t) g_{0} H_{\lambda}$ depend continuously upon $t g_{0} H_{\lambda}+(1-t) g_{1} H_{\mu} \in \Delta$ and $q^{\prime}\left(g_{1} A_{\mu}\right)=q^{\prime \prime}\left(g_{0} A_{\lambda}\right)$, we see that the continuity of $q^{\prime}$ and $q^{\prime \prime}$ implies the continuity of $q$. The equivariance of $q$ is evident. Let us check that $q(y) \in \mathcal{P}(n)$.

Indeed, since for each $t \in[0,1 / 2]$,

$$
g_{0} A_{\lambda} \subset q\left(t g_{0} H_{\lambda}+(1-t) g_{1} H_{\mu}\right) \subset B^{n},
$$

we infer that $q\left(t_{0} H_{\lambda}+(1-t) g_{1} H_{\mu}\right)$ belongs to $L_{0}(n)$. Further, for each $t \in[0,1 / 2]$, the contact set of $q\left(t g_{0} H_{\lambda}+(1-t) g_{1} H_{\mu}\right)$ is a subset of the contact set of $g_{0} A_{\lambda}$, and hence, it has an empty interior in the sphere $S^{n-1}$.

Analogously, for $t \in[1 / 2,1]$,

$$
g_{1} A_{\mu} \subset q\left(t g_{0} H_{\lambda}+(1-t) g_{1} H_{\mu}\right) \subset B^{n},
$$

which implies that $q\left(t g_{0} H_{\lambda}+(1-t) g_{1} H_{\mu}\right)$ belongs to $L_{0}(n)$. The contact set of $q\left(t g_{0} H_{\lambda}+(1-t) g_{1} H_{\mu}\right)$ is a subset of the contact set of $g_{1} A_{\mu}$, and hence, it also has an empty interior in the sphere $S^{n-1}$.

Thus, we have proved that for arbitrary $y \in \Gamma, q(y)$ belongs to $\mathcal{P}(n)$. 
By Lemma 4.4, there is a $G$-map $R: \mathcal{N}(\mathcal{U}) \rightarrow \mathcal{F}_{\infty}(\Gamma)$ satisfying conditions (1) and (2) of Lemma 4.4. Observe that for every $y \in \mathcal{N}(\mathcal{U}), R(y)$ is a finite collection of elements of $\Gamma$. Now we define $\Phi^{\prime}: \mathcal{N}(\mathcal{U}) \rightarrow \exp \mathbb{R}^{n}$ by

$$
\Phi^{\prime}(y)=\bigcup q(R(y))=\bigcup_{x \in R(y)} q(x), \quad y \in \mathcal{N}(\mathcal{U}) .
$$

Then $\Phi^{\prime}$ is well-defined, and by 23 , Corollary 5.3.7] it is continuous. The equivariance of $\Phi^{\prime}$ follows from that of $R$ and of $q$.

Let $\Phi(y)$ be the convex hull of the set $\Phi^{\prime}(y)$. Since $\Phi^{\prime}(y) \subset \Phi(y) \subset B^{n}$, we infer that $\Phi(y) \in L_{0}(n)$.

The continuity of $\Phi$ now follows from the continuity of $\Phi^{\prime}$ and of the convex hull operator (see [31] Theorem 2.7.4(iv)]).

Next, since $\Phi^{\prime}(y)$ is a finite union of bodies from $\mathcal{P}(n)$, its contact set, i.e., the intersection $\partial B^{n} \cap \partial \Phi^{\prime}(y)$, is the finite union of the contact sets of these bodies from $\mathcal{P}(n)$. Therefore, $\partial B^{n} \cap \partial \Phi^{\prime}(n)$ has an empty interior in $\partial B^{n}$. It remains to observe that $\Phi(y)$ and $\Phi^{\prime}(y)$ have the same contact set, and hence, $\Phi(y) \in \mathcal{P}(n)$.

Let us check that $\Phi\left(S t\left(g H_{\lambda}, \mathcal{N}(\mathcal{U})\right)\right) \subset V(g, \lambda)$ for all $g \in G, \lambda \in \Lambda$. Here we need the following:

Claim. Let $O(X, a), a>0$, be the open $a$-ball in $\mathcal{B}(n)$ centered at $X \in \mathcal{B}(n)$. If $y=t g_{0} H_{\lambda}+(1-t) g_{1} H_{\mu}$ is a point of the 1-cell $\left\langle g_{0} H_{\lambda}, g_{1} H_{\mu}\right\rangle$ such that the corresponding sets $g_{0} A_{\lambda}$ and $g_{1} A_{\mu}$ belong to $O(X, a)$, then $q(y) \in O(X, a) \cap L_{0}(n)$.

Proof of the Claim. First of all we observe that $O(X, a)$ is always a convex set in $\mathcal{B}(n)$, i.e., if $Y, Z \in O(X, a)$, then for every $t \in[0,1]$ the convex body $t X+(1-t) Z$ belongs to $O(X, a)$.

Hence, $s_{1}(y)=t g_{0} A_{\lambda}+(1-t) A_{\mu} \in O(X, a)$, due to the convexity of $O(X, a)$. One also has $s_{2}(y)=g_{0} A_{\lambda} \in O(X, a)$.

On the other hand, according to ([31, Theorem 2.7.4(iv)]), the convex hull operator is non-expansive. So for $0 \leq t \leq 1 / 2$ we have

$$
\begin{gathered}
d_{H}\left(q^{\prime}\left((1-2 t) g_{0} H_{\lambda}+2 t g_{1} H_{\mu}\right), X\right) \\
\left.\leq d_{H}\left(\left((1-2 t) g_{0} A_{\lambda}+2 t g_{1} A_{\mu}\right) \cup g_{0} A_{\lambda}\right), X\right)<a .
\end{gathered}
$$

Similarly, for $1 / 2 \leq t \leq 1$ we have

$$
\begin{gathered}
d_{H}\left(q^{\prime \prime}\left((2 t-1) g_{1} H_{\mu}+(2-2 t) g_{0} H_{\lambda}\right), X\right) \\
\left.\leq d_{H}\left(\left((2 t-1) g_{1} H_{\mu}+(2-2 t) g_{0} H_{\lambda}\right) \cup g_{1} A_{\mu}\right), X\right)<a .
\end{gathered}
$$

Therefore, $q(y)$ belongs to $O(X, a)$. Since $q(y) \in L_{0}(n)$, we infer finally that $q(y) \in O(X, a) \cap L_{0}(n)$, which completes the proof of the claim.

Now assume that $y \in S t\left(g H_{\lambda}, \mathcal{N}(\mathcal{U})\right)$ is an arbitrary point. We have to show that $\Phi(y) \in V(g, \lambda)$.

Let

$$
\tau=\left\langle g_{0} H_{\lambda_{0}}, \ldots, g_{n} H_{\lambda_{n}}\right\rangle
$$

be the carrier of $y$, where $g_{0}=g$ and $\lambda_{0}=\lambda$. Since $g_{0} S_{\lambda_{0}} \cap \cdots \cap g_{n} S_{\lambda_{n}} \neq \emptyset$, it follows from (5.1) that

$$
g_{i} X_{\lambda_{i}} \in S t\left(g_{0} S_{\lambda_{0}}, \mathcal{U}\right) \subset V\left(g_{0}, \lambda_{0}\right) / 2 \quad \text { for all } 0 \leq i \leq n .
$$


Let $V\left(g_{0}, \lambda_{0}\right)$ be the intersection $L_{0}(n) \cap O(Y, \varepsilon / 2)$ for some $Y \in L_{0}(n)$. It follows from (5.2) that

$$
d_{H}\left(g_{\lambda_{i}} X_{\lambda_{i}}, Y\right)<\varepsilon / 4 \quad \text { for all } 0 \leq i \leq n
$$

Since

$$
d_{H}\left(g_{i} A_{\lambda_{i}}, g_{i} X_{\lambda_{i}}\right)=d_{H}\left(A_{\lambda_{i}}, X_{\lambda_{i}}\right)<\varepsilon / 4
$$

it follows from (5.3) that

$$
g_{i} A_{\lambda_{i}} \in O(Y, \varepsilon / 2) \quad \text { for all } \quad 0 \leq i \leq n .
$$

By Lemma 4.4, $R(y)$ belongs to the 1 -skeleton of the $n$-cell $\left\langle g_{0} H_{\lambda_{0}}, \ldots, g_{n} H_{\lambda_{n}}\right\rangle$, i.e., to the union

$$
\bigcup_{i, j=0}^{n}\left\langle g_{i} H_{\lambda_{i}}, g_{j} H_{\lambda_{j}}\right\rangle .
$$

Now, it follows from (5.4) and the above claim that the image under $q$ of each 1-cell $\left\langle g_{i} H_{\lambda_{i}}, g_{j} H_{\lambda_{j}}\right\rangle, 0 \leq i, j \leq n$, lies in $O(Y, \varepsilon / 2)$. Since

$$
\Phi^{\prime}(y)=\bigcup_{x \in R(y)} q(x),
$$

we infer that $\Phi^{\prime}(y)$ also lies in $O(Y, \varepsilon / 2)$. Since $\Phi(y)$ is the convex hull of $\Phi^{\prime}(y)$ and $Y$ is convex, the inequality $d_{H}(\operatorname{conv} A$, conv $B) \leq d_{H}(A, B)$ (see 31, Theorem 2.7.4(iv)]) implies that $d_{H}(\Phi(y), Y)<\varepsilon / 2$, i.e., $\Phi(y) \in O(Y, \varepsilon / 2)$. Consequently,

$$
\Phi(y) \in O(Y, \varepsilon / 2) \cap L_{0}(n)=V\left(g_{0}, \lambda_{0}\right)=V(g, \lambda),
$$

as required.

The second claim of Lemma 5.2 now follows immediately from the first one.

Indeed, for any $A \in L_{0}(n)$ there is $\lambda \in \Lambda$ such that $\varphi_{\lambda}(A)>0$. Then $A \in G\left(S_{\lambda}\right)$, and hence, $A \in g S_{\lambda}$ for some $g \in G$. It then follows from the definition of the map $p: L_{0}(n) \rightarrow \mathcal{N}(\mathcal{U})$ that $p(A) \in S t\left(g H_{\lambda}, \mathcal{N}(\mathcal{U})\right.$ ) (see the proof of Lemma 4.2). By the first statement, $\Phi(p(A)) \in V(g, \lambda)$. Since $A \in g S_{\lambda} \subset V(g, \lambda)$, we see that $A$ and $\Phi(p(A))$ are $\varepsilon$-close. This means that the map $\Phi p$ is $\varepsilon$-close to the identity map of $L_{0}(n)$, which completes the proof.

Let $(X, d)$ be a metric space with a geodesic (or convex) metric $d$, i.e., for any two points $x, y \in X$ there is an isometry $\iota:[0, d(x, y)] \rightarrow X$ such that $\iota(0)=x$ and $\iota(d(x, y))=y$. For any element $A \in \exp X$ the generalized closed $r$-ball centered at $A$ is the set $A_{r}=\{x \in X \mid d(x, A) \leq r\}$. If $X=\mathbb{R}^{n}$ and $A \in \mathcal{B}(n)$, then $A_{r}$ is just the parallel body $A+r B^{n}$, and hence, in this case, $A_{r}$ is a compact, convex, centrally symmetric body.

Lemma 5.3. Let $(X, d)$ be a metric space with a geodesic (or convex) metric d. Then for any two elements $A, C \in \exp X$ and any two numbers $r, s>0$, the following hold:

(1) $d_{H}\left(A_{r}, B_{r}\right) \leq d_{H}(A, B)$,

(2) $d_{H}\left(A_{r}, A_{s}\right) \leq|r-s|$.

Proof. Since $d$ is a convex metric, the first claim follows from [19. Proposition 10.5]. The second one follows from the property $\left(A_{p}\right)_{q}=A_{p+q}$ for any two nonnegative reals $p$ and $q$ (see [25, p. 38, Exercise 0.65.3(c)]). 
Lemma 5.4. For each $\varepsilon>0$ there exist $O(n)$-equivariant maps $f_{\varepsilon}, h_{\varepsilon}: L_{0}(n) \rightarrow$ $L_{0}(n), \varepsilon$-close to the identity map of $L_{0}(n)$, such that the images of $f_{\varepsilon}$ and $h_{\varepsilon}$ are disjoint.

Proof. Define a continuous map $\gamma: L_{0}(n) \rightarrow \mathbb{R}$ by the rule

$$
\gamma(A)=(1 / 2) \min \left\{d_{H}\left(B^{n}, A\right), \varepsilon\right\} \quad \text { for every } A \in L_{0}(n) .
$$

Let $f_{\epsilon}$ be just the closed $\gamma(A)$-neighborhood of $A$ in $B^{n}$, i.e.,

$$
f_{\varepsilon}(A)=A_{\gamma(A)} .
$$

By the choice of $\gamma(A)$, the set $f_{\varepsilon}(A)$ is different from $B^{n}$, and since $A \subset f_{\varepsilon}(A)$, we see that $f_{\varepsilon}(A) \in L_{0}(n)$. It is clear from the construction that $f_{\varepsilon}$ is $\varepsilon$-close to the identity map of $L_{0}(n)$.

Let us check the continuity of $f_{\epsilon}$. We have

$$
d_{H}\left(f_{\varepsilon}(A), f_{\varepsilon}(C)\right)=d_{H}\left(A_{\gamma(A)}, C_{\gamma(C)}\right) \leq d_{H}\left(A_{\gamma(A)}, A_{\gamma(C)}\right)+d_{H}\left(A_{\gamma(C)}, C_{\gamma(C)}\right) .
$$

But by Lemma 5.3

$$
d_{H}\left(A_{\gamma(A)}, A_{\gamma(C)}\right) \leq|\gamma(A)-\gamma(C)| \quad \text { and } \quad d_{H}\left(A_{\gamma(C)}, C_{\gamma(C)}\right) \leq d_{H}(A, C) .
$$

Thus,

$$
d_{H}\left(f_{\varepsilon}(A), f_{\varepsilon}(C)\right) \leq|\gamma(A)-\gamma(C)|+d_{H}(A, C) .
$$

Now the continuity of $f_{\varepsilon}$ follows from that of $\gamma$. The $O(n)$-equivariance of $f_{\varepsilon}$ is immediate from the invariance of the metric $d$.

Next, we define the map $h_{\varepsilon}: L_{0}(n) \rightarrow L_{0}(n)$ to be the composition $\Phi p$ from Lemma 5.2. Then $f_{\varepsilon}(A) \neq h_{\varepsilon}(C)$ for all $A, C \in L_{0}(n)$, since the contact set of $f_{\varepsilon}(A)$ has a nonempty interior in the boundary sphere $S^{n-1}=\partial B^{n}$ while the contact set of $h_{\varepsilon}(C)$ has an empty interior in $S^{n-1}$ (this is because $h_{\varepsilon}(C) \in \mathcal{P}(n)$ ).

Lemma 5.5. There is an $O(n)$-equivariant strong deformation retraction $\left(f_{t}\right)$ of $L(n)$ to its point $B^{n}$ such that $f_{t}: L(n) \rightarrow L(n)$ is an $O(n)$-isovariant map for all $0<t \leq 1$. In particular, $f_{t}(A) \neq\left\{B^{n}\right\}$ for all $0<t \leq 1$.

Proof. For each $A \in L(n)$ and $0 \leq t \leq 1$, write

$$
f_{t}(A)=(1-t) B^{n}+t A .
$$

Proof of Theorem 1.3. Since, by Theorem 2.4 $L(n)$ is an $O(n)$-AR, we infer that it is also an $H$-AR (see, e.g., 30|). Therefore, by Theorem 2.7, $L(n) / H$ is an AR. Since $L_{0}(n) / H$ is an open subset of $L(n) / H$, it is a locally compact ANR. Now, in order to prove that $L_{0}(n) / H$ is a $Q$-manifold it suffices, according to Toruńczyk 's Characterization Theorem [28, to check that for every $\varepsilon>0$ there are continuous maps $f_{\varepsilon}^{\prime}, h_{\varepsilon}^{\prime}: L_{0}(n) / H \rightarrow L_{0}(n) / H, \varepsilon$-close to the identity map of $L_{0}(n) / H$, such that the images of $f_{\varepsilon}^{\prime}$ and $h_{\varepsilon}^{\prime}$ are disjoint. But this is immediate from Lemma 5.4. if we take for $f_{\varepsilon}^{\prime}$ and $h_{\varepsilon}^{\prime}$ the maps induced by $f_{\varepsilon}$ and $h_{\varepsilon}$, respectively.

The $[0,1)$-stability of $L_{0}(n) / H$ follows from Lemma 5.5 which yields that the space $L_{0}(n) / H$ possesses an obvious proper deformation (preimage of each compact set is compact) to infinity:

$$
\left(L_{0}(n) / H\right) \times[0,1) \rightarrow L_{0}(n) / H .
$$


Hence, by a result of R. Y. T. Wong [36], $L_{0}(n) / H$ is homeomorphic to its product with the half-open interval $[0,1)$, i.e., $L_{0}(n) / H$ is $[0,1)$-stable. This completes the proof.

The following lemma for $n=2$ was proved in [9]:

Lemma 5.6. For each closed subgroup $K \subset O(n)$ acting non-transitively on $S^{n-1}$, and each $\varepsilon>0$, there is a $K$-equivariant map $h_{\varepsilon}: L(n) \rightarrow L_{0}(n)$, $\varepsilon$-close to the identity map of $L(n)$. In particular, $h_{\varepsilon}(L(n)[K]) \subset L_{0}(n)[K]$.

Proof. Let $r: \mathcal{B}(n) \rightarrow L(n)$ be the $O(n)$-equivariant retraction from Theorem 2.3 Because $L(n)$ is compact (Theorem 2.4), one can find a real $0<\delta<\varepsilon / 2$ such that $d_{H}(r(A), A)<\varepsilon / 2$ for all $A$ belonging to the $\delta$-neighborhood of $L(n)$ in $\mathcal{B}(n)$, where $d_{H}$ denotes the Hausdorff metric on $\mathcal{B}(n)$.

Fix a centrally symmetric, convex polyhedron $P \subset \mathbb{R}^{n}$ with a nonempty interior, inscribing $B^{n}$, i.e., $P \subset B^{n}$ and all the vertices $p_{1}, \ldots, p_{k}$ of $P$ lie on the unit sphere $S^{n-1}=\partial B^{n}$. Then the convex hull

$$
R=\operatorname{conv}\left(K\left(p_{1}\right) \cup \cdots \cup K\left(p_{k}\right)\right)
$$

is a centrally symmetric, compact, convex, $K$-invariant subset of $\mathbb{R}^{n}$. Since it contains $P$, we see that $R$ has a nonempty interior, and hence, $R \in \mathcal{B}(n)$. We claim that the boundary $\partial R$ does not contain an $(n-1)$-dimensional elliptic domain, i.e., an open connected subset of some $(n-1)$-dimensional ellipsoid surface lying in $\mathbb{R}^{n}$. It suffices to show that none of the orbits $K\left(p_{i}\right), i=1, \ldots, k$, contains an $(n-1)$-dimensional elliptic domain. Assume the contrary, that $K\left(p_{i}\right)$ contains an $(n-1)$-dimensional elliptic domain. Since $K\left(p_{i}\right)$ lies on the sphere $S^{n-1}$, then this domain should be in fact a domain of the sphere $S^{n-1}$. Since $K\left(p_{i}\right)$ is homogeneous and compact, we conclude that there are finitely many open subsets $V_{1}, \ldots, V_{n}$ of $K\left(p_{i}\right)$ such that $K\left(p_{i}\right)=V_{1} \cup \cdots \cup V_{n}$, where each $V_{j}$ is homeomorphic to $V$. Next, by the Domain Invariance Theorem (see, e.g., [27, Ch. 4, Section 7, Theorem 16]), each $V_{j}$ should be open in the sphere $S^{n-1}$, and hence, the union $V_{1} \cup \cdots \cup V_{n}$ is open in $S^{n-1}$. But $V_{1} \cup \cdots \cup V_{n}$ is also closed in $S^{n-1}$, because it is equal to $K\left(p_{i}\right)$. Now, by connectedness of $S^{n-1}$, it then follows that $K\left(p_{i}\right)$ is the whole sphere $S^{n-1}$. Consequently, $K$ acts transitively on the unit sphere $S^{n-1}$, a contradiction. The claim is proved.

Now, let $a$ be the distance of the origin from the boundary of $R$, and $T=$ $(1 / a) R$. Then $T$ is a $K$-invariant, centrally symmetric, compact, convex body that circumscribes the unit ball $B^{n}$, i.e., $B^{n} \subset T$ and the boundaries $\partial T$ and $\partial B^{n}$ have a nonempty intersection.

Setting

$$
h^{\prime}(A)=A \cap(1-\delta) T
$$

we obtain a map $h^{\prime}: L(n) \rightarrow \mathcal{B}(n)$. Since $T$ is a $K$-fixed point of $\mathcal{B}(n)$, we see that $h^{\prime}$ is $K$-equivariant.

Continuity of $h^{\prime}$ is evident.

Since $d_{H}\left(A, A \cap(1-\delta) B^{n}\right) \leq \delta$ and $A \cap(1-\delta) B^{n} \subset h^{\prime}(A) \subset A$, we conclude that $d_{H}\left(A, h^{\prime}(A)\right) \leq \delta$. In particular, $h^{\prime}$ is $(\varepsilon / 2)$-close to the inclusion $L(n) \hookrightarrow \mathcal{B}(n)$.

We claim that $h^{\prime}(A)$ is not an ellipsoid for each $A \in L(n)$. Indeed, if $A \subset(1-\delta) T$, then $A \neq B^{n}$, since $T$ circumscribes $B^{n}$ and $1-\delta<1$. On the other hand, $h^{\prime}(A)=A$ in this case, and hence, $h^{\prime}(A)$ is not an ellipsoid. If $A$ is not contained in $(1-\delta) T$, then the boundary of $h^{\prime}(A)$ contains a domain lying in the boundary 
of $(1-\delta) T$. Since $(1-\delta) T=((1-\delta) / a) R$ and since the boundary of $R$ does not contain an $(n-1)$-dimensional elliptic domain (as shown above), we conclude that the boundary of $(1-\delta) T$ does not contain an $(n-1)$-dimensional elliptic domain as well. Thus, $h^{\prime}(A)$ is not an ellipsoid, and the claim is proved.

Since $r\left(h^{\prime}(A)\right)$ and $h^{\prime}(A)$ have the same $G L(n)$-orbit, we conclude that $r\left(h^{\prime}(A)\right)$ $\neq B^{n}$ for each $A \in L(n)$. Since $r$ is $O(n)$-equivariant and $h^{\prime}$ is $K$-equivariant, denoting by $h_{\varepsilon}$ the composition $r h^{\prime}$, we obtain a $K$-equivariant map $h_{\varepsilon}: L(n) \rightarrow$ $L_{0}(n), \varepsilon$-close to the identity map of $L(n)$.

Proof of Theorem 1.4 For the first claim it suffices to show that $L_{0}(n) / K$ is homeomorphic to $Q_{0}$, the Hilbert cube with a removed point. By Theorem $1.3 L_{0}(n) / K$ is a $[0,1)$-stable $Q$-manifold. On the other hand, $Q_{0}$ is a contractible $[0,1)$-stable $Q$-manifold. Therefore, according to a result of T. A. Chapman [12, Theorem 21.2], it remains only to check that $L_{0}(n) / K$ and $Q_{0}$ are homotopically equivalent, i.e., that $L_{0}(n) / K$ is contractible.

According to Theorem 2.4 $L(n)$ is an $O(n)$-AR, which in turn implies that $L(n) \in K$-AR (see, e.g., 30]). Then, by Theorem $2.7 L(n) / K$ is an AR, and hence, is contractible. It follows from Lemma 5.6 that the singular point $\left\{B^{n}\right\}$ is a $Z$-set in the $K$-orbit space $L(n) / K$, and hence, according to [18], $L(n) / K$ and $L_{0}(n) / K$ have the same homotopy type. Since $L(n) / K$ is contractible, we see that $L_{0}(n) / K$ is contractible too.

For the second claim, it suffices to show that $L_{0}(n)[K]$ is homeomorphic to $Q_{0}$. First we show that $L_{0}(n)[K]$ is a $[0,1)$-stable $Q$-manifold. Indeed, by Theorem 2.4 $L(n)$ is an $O(n)$-AR, and hence, $L(n)[K]$ is an AR [2, Theorem 7]. It then follows that $L_{0}(n)[K]$ is a locally compact ANR. Now by Toruńczyk's Characterization Theorem [28], $L_{0}(n)[K]$ is a $Q$-manifold if we observe that the equivariant maps $f_{\varepsilon}$ and $h_{\varepsilon}$ from Lemma 5.4 take $L_{0}(n)[K]$ into itself. The $[0,1)$-stability of $L_{0}(n)[K]$ can be proved like that of $L_{0}(n) / K$ in Theorem 1.3 . Indeed, Lemma 5.5yyields that the space $L_{0}(n)[K]$ possesses an obvious proper deformation to infinity

$$
\left(L_{0}(n)[K]\right) \times[0,1) \rightarrow L_{0}(n)[K] .
$$

Hence, by the result of R. Y. T. Wong [36], $L_{0}(n) / H$ is $[0,1)$-stable.

Let us show that $L_{0}(n)[K]$ is contractible. Since $L_{0}(n)[K] \neq \emptyset$, according to Lemma [5.6, the singular point $\left\{B^{n}\right\}$ is a $Z$-set in $L(n)[K]$. It then follows from [18] that $L(n)[K]$ and $L_{0}(n)[K]$ have the same homotopy type. But since $L(n)[K]$ is an AR, it is contractible, and hence, $L_{0}(n)[K]$ is contractible too. Since $Q_{0}$ also is a $[0,1)$-stable contractible $Q$-manifold, it only remains to apply the above-quoted result of T. A. Chapman to the $Q$-manifolds $L_{0}(n)[K]$ and $Q_{0}$. This completes the proof.

\section{Proof of Theorem 1.6}

We first prove the following fact.

Lemma 6.1. $L_{0}(n)$ and $\Pi_{0}(n)$ have the same $O(n)$-homotopy type.

Proof. By [9] Lemma 4], there is an isovariant map $f: L_{0}(n) \rightarrow \Pi(n)$, yielding that the image of $f$ lies, in fact, in $\Pi_{0}(n)$. Hence, the result follows from the following: Claim. Every $O(n)$-equivariant map $f: L_{0}(n) \rightarrow \Pi_{0}(n)$ is an $O(n)$-homotopy equivalence. 
To prove this claim we apply the James-Segal Theorem 2.5. In our case $G=O(n)$, $T=L_{0}(n)$ and $Z=\Pi_{0}(n)$.

By Theorem [2.4, $L(n) \in O(n)$-AR, implying that $L_{0}(n) \in O(n)$-ANR. Let $K \subset$ $O(n)$ be a closed subgroup such that $L_{0}(n)[K] \neq \emptyset$. As we have seen above in the proof of Theorem [1.4 $L_{0}(n)[K]$ is contractible. Besides, it follows from the equivariance of $f$ that $L_{0}(n)[K] \subset \Pi_{0}(n)[K]$, and so $\Pi_{0}(n)[K] \neq \emptyset$.

On the other hand, since $O(n) / H_{i} \in O(n)$-ANR [26, p. 27], we infer that Cone $\left(O(n) / H_{i}\right) \in O(n)$-AR (see [9, Lemma 3]). Consequently, $Q\left(H_{i}\right) \in O(n)$ AR, $i \geq 1$, and hence, $\Pi(n) \in O(n)$-AR, implying $\Pi_{0}(n) \in O(n)$-ANR. Thus, it remains only to show that for each closed subgroup $K \subset O(n)$ with $\Pi_{0}(n)[K] \neq \emptyset$, $\Pi_{0}(n)[K]$ is contractible and, at the same time, $L_{0}(n)[K] \neq \emptyset$. We will show that in fact $\Pi(n)[K]$ is a Hilbert cube, implying the contractibility of $\Pi_{0}(n)[K]$. Indeed, it is not hard to see that if $\Pi_{0}(n)[K] \neq \emptyset$, then there is an orbit type $\left(H_{i}\right)$ such that $\left(O(n) / H_{i}\right)[K] \neq \emptyset$. This implies that $(K) \preceq\left(H_{i}\right)$, and so, $K \subset g H_{i} g^{-1}$ for some $g \in O(n)$. But $H_{i}$ occurs in $L_{0}(n)$ as a stabilizer. So there exists a body $A \in L_{0}(n)$ such that $O(n)_{A}=H_{i}$. Consequently, $K \subset O(n)_{g A}$. Since $g A \in L_{0}(n)$, we see that $L_{0}(n)[K] \neq \emptyset$. Next we have

$$
\Pi(n)[K]=\prod_{i=1}^{\infty}\left(Q\left(H_{i}\right)[K]\right) .
$$

Since $O(n) / H_{i} \in O(n)$-ANR, we see that $\left(O(n) / H_{i}\right)[K]$ is a nonempty ANR. Consequently,

$$
\left(\operatorname{Cone}\left(O(n) / H_{i}\right)\right)[K]=\operatorname{Cone}\left(\left(O(n) / H_{i}\right)[K]\right)
$$

is a nondegenerate AR. Hence, according to a result of West [32], the countable product

$$
Q\left(H_{i}\right)[K]=\left(\operatorname{Cone}\left(O(n) / H_{i}\right)[K]\right)^{\infty}
$$

is a Hilbert cube. This implies that $\prod_{i=1}^{\infty}\left(Q\left(H_{i}\right)[K]\right)$ is a Hilbert cube, and hence, $\Pi_{0}(n)[K]$ is contractible. By applying the above-mentioned James-Segal Theorem, we complete the proof.

Proof of Theorem 1.6. Since by Theorem[1.3, $L_{0}(n) / H$ is a $[0,1)$-stable $Q$-manifold, according to Chapman's theorem [12, Theorem 21.2], it remains only to prove that $\Pi_{0}(n) / H$ is a $[0,1)$-stable $Q$-manifold of the same homotopy type as $L_{0}(n) / H$.

The fact that $\Pi_{0}(n) / H$ is a $Q$-manifold is proved in [9] Theorem A1]. Its [0,1)stability follows from Wong's theorem [36] if we observe that $\Pi_{0}(n) / H$ possesses a proper deformation to infinity:

$$
\left(\Pi_{0}(n) / H\right) \times[0,1) \rightarrow \Pi_{0}(n) / H .
$$

Indeed, this follows easily from the conic structure of $\Pi(n)$.

Finally, that $\Pi_{0}(n) / H$ and $L_{0}(n) / H$ have the same homotopy type follows immediately from Lemma 6.1.

\section{Proof of Theorem 1.9 and Reduction of Conjecture 1.8}

We start with the following lemma. 
Lemma 7.1. Let $G$ be a compact group, $N \subset G$ a closed, normal subgroup and $X$ a $G$-ANR (resp., a $G$-AR). Then the $N$-fixed point set $X[N]$ is a $G$-ANR (resp., a $G-A R)$ as well.

Proof. According to [5. Corollary 5], there is a normed linear space $L$ such that $X$ can be embedded as a closed invariant subspace into $Z=C(G, L)$, the normed linear $G$-space of all continuous maps $f: G \rightarrow L$ endowed with the sup-norm and with the action $g f$ of $G$ defined by

$$
(g f)(x)=f(x g) ; \quad f \in C(G, L) \quad g, x \in G .
$$

Then there is a $G$-retraction $r: U \rightarrow X$ for some open $G$-neighborhood $U$ of $X$ in $Z$ (resp., $U=Z$ ). Therefore, it suffices to prove that $Z[N]$ is a $G$-AR. One easily sees that $Z[N]=C(G / N, L)$, where the $G$-action $g \phi$ on $C(G / N, L)$ is defined by

$$
(g \phi)(x N)=\phi(x g N), \quad \text { for } \quad \phi \in C(G / N, L) \quad \text { and } \quad g \in G, x N \in G / N .
$$

Now $C(G / N, L)$ is a $G$-AR by [5, Theorem 8].

Let $\operatorname{sexp} S^{n}$ be the subspace of $\exp S^{n}$ consisting of all centrally symmetric sets $A \subset S^{n}$, i.e., $A=-A$. By $\operatorname{sexp}_{0} S^{n}$ we will denote the complement $\left(\operatorname{sexp} S^{n}\right) \backslash\left\{S^{n}\right\}$. Evidently, $\operatorname{sexp} S^{n}$ is an $O(n)$-invariant subset of $\exp S^{n}$.

The next lemma is immediate from Theorem 3.1 and Lemma 7.1 if we observe that $\operatorname{sexp} S^{n-1}=\left(\exp S^{n-1}\right)[N]$ with $N=\left\{1_{\mathbb{R}^{n}},-1_{\mathbb{R}^{n}}\right\}$ :

Lemma 7.2. $\operatorname{sexp} S^{n-1}$ is an $O(n)-A R$.

Lemma 7.3. There exists an $O(n)$-equivariant map $f: \operatorname{sexp}_{0} S^{n-1} \rightarrow L_{0}(n)$.

Proof. For every $A \in \operatorname{sexp}_{0} S^{n-1}$, let

$$
\varphi(A)=\operatorname{conv}(A \cup B(0,1 / 2)),
$$

where $B(0,1 / 2)$ is the closed $1 / 2$-ball in $\mathbb{R}^{n}$ centered at the origin, and conv stands for the convex hull. Clearly, $\varphi$ is a well-defined, continuous, $O(n)$-equivariant map of $\operatorname{sexp}_{0} S^{n-1}$ into $\mathcal{B}(n)$. Furthermore, $\varphi(A)$ is not an ellipsoid because $A \neq S^{n-1}$, implies that the boundary $\partial(\varphi(A))$ contains a nontrivial line segment. Now we set $f=r \varphi$, where $r: \mathcal{B}(n) \rightarrow L(n)$ is the $O(n)$-equivariant map from Theorem 2.3. Since $\varphi(A)$ is not an ellipsoid, and since $r$ preserves the $G L(n)$-orbit, we conclude that $f(A)$ is not the unit ball $B^{n}$; so $f(A) \in L_{0}(n)$. Since $r$ and $\varphi$ are $O(n)$-equivariant, so is $f$.

Lemma 7.4. Let $K \subset O(n)$ be a closed subgroup. Then the following conditions are equivalent:

(1) $K$ acts non-transitively on the sphere $S^{n-1}$,

(2) $\left(\exp _{0} S^{n-1}\right)[K] \neq \emptyset$,

(3) $\left(\operatorname{sexp}_{0} S^{n-1}\right)[K] \neq \emptyset$,

(4) $L_{0}(n)[K] \neq \emptyset$.

Proof. (1) $\Longrightarrow(2)$. If $K$ acts non-transitively, then there is a $K$-invariant proper subset $A \subset S^{n-1}$; so $A \in\left(\exp _{0} S^{n-1}\right)[K]$.

$(2) \Longrightarrow(3)$. If $A \in\left(\exp _{0} S^{n-1}\right)[K]$, then either

$$
(-A) \cup A \neq S^{n-1} \quad \text { or } \quad(-A) \cap A \neq \emptyset,
$$

and so at least one of the sets $(-A) \cup A$ and $(-A) \cap A$ belongs to $\left(\operatorname{sexp}_{0} S^{n-1}\right)[K]$. Thus, $\left(\operatorname{sexp}_{0} S^{n-1}\right)[K] \neq \emptyset$. 
$(3) \Longrightarrow(4)$ is immediate from Lemma 7.3 .

$(4) \Longrightarrow(1)$. If there is a body $A \in L_{0}(n)[K]$, then the contact set $A \cap S^{n-1}$ is a nonempty, $K$-invariant, proper subset of $S^{n-1}$. So the action of $K$ on $S^{n-1}$ is not transitive.

Lemma 7.5. Every $O(n)$-equivariant map $f: \operatorname{sexp}_{0} S^{n-1} \rightarrow L_{0}(n)$ is an $O(n)$ homotopy equivalence.

Proof. We are going to apply the James-Segal Theorem 2.5 with $G=O(n), T=$ $\operatorname{sexp}_{0} S^{n-1}$ and $Z=L_{0}(n)$. It follows from Lemma 7.2 that $\operatorname{sexp}_{0} S^{n-1} \in O(n)$ ANR. Since $L(n) \in O(n)$-AR (see Theorem 2.4), we see that also $L_{0}(n) \in O(n)$ ANR.

Let $K \subset O(n)$ be a closed subgroup. Then by Lemma $7.4,\left(\operatorname{sexp}_{0} S^{n-1}\right)[K] \neq \emptyset$ iff $L_{0}(n)[K] \neq \emptyset$. Let $L_{0}(n)[K] \neq \emptyset$ and $K^{\prime}=K \times\left\{1_{\mathbb{R}^{n}},-1_{\mathbb{R}^{n}}\right\}$. Since

$$
\left(\exp _{0} S^{n-1}\right)\left[K^{\prime}\right]=\left(\operatorname{sexp}_{0} S^{n-1}\right)[K] \neq \emptyset,
$$

the action of $K^{\prime}$ on $S^{n-1}$ is not transitive, i.e., $S^{n-1} / K^{\prime}$ is not a singleton. Since $\left(\exp S^{n-1}\right)\left[K^{\prime}\right]$ is homeomorphic to $\exp \left(S^{n-1} / K^{\prime}\right)$ and $S^{n-1} / K^{\prime}$ is a nondegenerate Peano continuum, by the Curtis-Schori-West Hyperspace Theorem (see, e.g., [23. $\S 8.4]), \exp \left(S^{n-1} / K^{\prime}\right)$, and hence $\left(\operatorname{sexp} S^{n-1}\right)[K]$, is a Hilbert cube. Consequently, $\left(\operatorname{sexp}_{0} S^{n-1}\right)[K]$, being a Hilbert cube with a removed point, is contractible.

According to Lemma [5.6, the singular point $\left\{B^{n}\right\}$ is a $Z$-set in $L(n)[K]$. Since $L(n)[K] \in \mathrm{AR}$, it then follows from [18] that $L(n)[K]$ and $L_{0}(n)[K]$ have the same homotopy type, and hence, $L_{0}(n)[K]$ is contractible too. Now, by applying the above mentioned James-Segal Theorem 2.5, we complete the proof.

Similarly, the following can be proved:

Lemma 7.6. Every $O(n)$-equivariant map $f: \operatorname{sexp}_{0} S^{n-1} \rightarrow \exp _{0} S^{n-1}$ is an $O(n)$-homotopy equivalence.

Proof. By Lemma [7.4 for any closed subgroup $K \subset O(n)$ one has

$$
\left(\operatorname{sexp}_{0} S^{n-1}\right)[K] \neq \emptyset \Longleftrightarrow\left(\exp _{0} S^{n-1}\right)[K] \neq \emptyset
$$

As in the proof of Lemma [7.5, $\left(\operatorname{sexp}_{0} S^{n-1}\right)[K]$, as well as $\left(\exp _{0} S^{n-1}\right)[K]$, is contractible whenever $\left(\exp _{0} S^{n-1}\right)[K] \neq \emptyset$ (or equivalently, $\left.\left(\operatorname{sexp}_{0} S^{n-1}\right)[K] \neq \emptyset\right)$. Now apply the James-Segal Theorem 2.5

Lemma 7.3 and Lemma 7.5 have the following immediate consequence.

Corollary 7.7. $\operatorname{sexp}_{0} S^{n-1}$ and $L_{0}(n)$ have the same $O(n)$-homotopy type.

Analogously, Lemma 7.6 implies the following.

Corollary 7.8. The natural inclusion $\operatorname{sexp}_{0} S^{n-1} \hookrightarrow \exp _{0} S^{n-1}$ is an $O(n)$-homotopy equivalence.

Proof of Theorem 1.9. Immediate from Corollaries 7.7 and 7.8 .

Our final result reduces Conjecture 1.8 to an easier one:

Theorem 7.9. For each closed subgroup $H \subset O(n)$, the two $H$-orbit spaces $L_{0}(n) / H$ and $\left(\exp _{0} S^{n-1}\right) / H$ are homeomorphic iff $\left(\exp _{0} S^{n-1}\right) / H$ is a $Q$-manifold.

For the proof we need the following fact. 
Lemma 7.10. There exists an $O(n)$-equivariant strong deformation retraction $\left(f_{t}\right)$ of $\exp S^{n-1}$ to its $O(n)$-fixed point $\left\{S^{n-1}\right\}$ such that $f_{t}(A) \neq\left\{S^{n-1}\right\}$ for all $0<$ $t \leq 1$ and $A \in \exp _{0} S^{n-1}$.

Proof. Observe that the usual spherical metric $d$ on $S^{n-1}$ is $O(n)$-invariant and convex. For each $A \in \exp S^{n-1}$ and $0 \leq t \leq 1$, write

$$
f_{t}(A)=\left\{x \in S^{n-1} \mid d(x, A) \leq(1-t) d_{H}\left(S^{n-1}, A\right)\right\} .
$$

Due to the convexity of $d$ this homotopy is continuous; it is also equivariant, since $d$ and $d_{H}$ are invariant. Other required properties of $\left(f_{t}\right)$ are evident.

Proof of Theorem 7.9. Since by Theorem 1.3, $L_{0}(n) / H$ is a $Q$-manifold, only the "if" part requires a proof.

So, assume that $\left(\exp _{0} S^{n-1}\right) / H$ is a $Q$-manifold. It follows from Theorem 1.9 that

$$
\left(\exp _{0} S^{n-1}\right) / H \quad \text { and } \quad L_{0}(n) / H
$$

have the same homotopy type. Moreover, by Theorem $1.3, L_{0}(n) / H$ is a $[0,1)$ stable $Q$-manifold. Therefore, according to Chapman's theorem [12, Theorem 21.2], it only remains to see that $\left(\exp _{0} S^{n-1}\right) / H$ is $[0,1)$-stable too. Indeed, Lemma 7.10 yields that $\left(\exp _{0} S^{n-1}\right) / H$ possesses an obvious proper deformation to infinity:

$$
\left(\left(\exp _{0} S^{n-1}\right) / H\right) \times[0,1) \rightarrow\left(\exp _{0} S^{n-1}\right) / H .
$$

Now Wong's result [36] implies that $\left(\exp _{0} S^{n-1}\right) / H$ is $[0,1)$-stable, and this completes the proof.

Acknowledgement. The author is grateful to the referee for helpful suggestions and remarks which led to a number of improvements of the text.

\section{REFERENCES}

1. H. Abels, Parallelizability of proper actions, global $K$-slices and maximal compact subgroups, Math. Ann. 212 (1974), 1-19. MR 51:11460

2. S. A. Antonyan, Retracts in categories of $G$-spaces, Izvestiya Akad. Nauk Arm. SSR. Ser. Matem. 15 (1980), 365-378; English transl. in: Soviet J. Contemp. Math. Anal. 15 (1980), 30-43. MR 82f:54027

3. S. A. Antonyan, Equivariant generalization of Dugundji's theorem, Mat. Zametki 38 (1985), 608-616; English transl. in: Math. Notes 38 (1985), 844-848. MR 87a:54053

4. S. A. Antonyan, An equivariant theory of retracts, in: Aspects of Topology (In Memory of Hugh Dowker), 251-269, London Math. Soc. Lecture Note Ser. 93, Cambridge Univ. Press, Cambridge, 1985. MR 87e:54090

5. S. A. Antonian, Equivariant embeddings into $G$-AR's, Glasnik Matematički 22 (42) (1987), 503-533. MR 89k:54041

6. S. A. Antonyan, Retraction properties of an orbit space, Matem. Sbornik 137 (1988), 300-318; English transl. in: Math. USSR Sbornik 65 (1990), 305-321. MR 89k:54042

7. S. A. Antonyan, Retraction properties of a space of orbits, II, Russian Math. Surv. 48 (1993), 156-157. MR 95b:54023

8. S. A. Antonyan, The Banach-Mazur compacta are absolute retracts, Bull. Acad. Polon. Sci. Ser. Math. 46 (1998), 113-119. MR 99d:54020

9. S. A. Antonyan, The topology of the Banach-Mazur compactum, Fund. Math. 166, no. 3 (2000), 209-232. MR 2001k:57026

10. S. Banach, Théorie des Opérations Linéaires, Monografje Matematyczne, Warszawa, 1932. MR 97d:01035 (reprint)

11. G. Bredon, Introduction to compact transformation groups, Academic Press, New YorkLondon, 1972. MR 54:1265 
12. T. A. Chapman, Lectures on Hilbert cube manifolds, C. B. M. S. Regional Conference Series in Math., 28, Amer. Math. Soc., Providence, RI, 1975. MR 54:11336

13. D. W. Curtis, Hyperspaces of noncompact metric spaces, Compositio Math. 40 (1980), 139152. MR 81c:54009

14. D. W. Curtis, Boundary sets in the Hilbert cube, Topol. Appl. 20 (1985), 201-221. MR 87d:57014

15. J. Dugundji, Topology, Allyn and Bacon Inc., Boston, 1966. MR 33:1824

16. P. Fabel, The Banach-Mazur compactum $Q(2)$ is an absolute retract, in: Topology and Applications (International Topological Conference dedicated to P. S. Alexandroff's 100th birthday, Moscow, May 27-31, 1996), p. 57, Moscow, 1996.

17. R. E. Heisey and J. E. West, Orbit spaces of the hyperspace of a graph which are Hilbert cubes, Colloq. Math. 56 (1988), 59-69. MR 90a:57024

18. D. W. Henderson, Z-sets in ANR's, Trans. Amer. Math. Soc. 213 (1975), 205-215. MR 52:11830

19. A. Illanes and S. B. Nadler Jr., Hyperspaces. Fundamentals and Recent Advances, Marcel Dekker, Inc., New York-Basel, 1999. MR 99m:54006

20. I. M. James and G. B. Segal On equivariant homotopy theory, Lecture Notes in Math. 788 (1980), 316-330. MR 82f:55014

21. F. John. Extremum problems with inequalities as subsidiary conditions, in: F. John, Collected papers, 2 (ed. by J. Moser), 543-560, Birkhäuser, 1985. MR 10:719b MR 87f:01107

22. T. Matumoto, On G-CW complexes and a theorem of J.H.C. Whitehead, J. Fac. Sci. Univ. Tokyo Sect. I A Math. 18 (1971), 363-374. MR 49:9842

23. J. van Mill, Infinite-dimensional topology. Prerequisites and Introduction, North-Holland Publ. Co., Amsterdam-New York-Oxford-Tokyo, 1989. MR 90a:57025

24. J. Milnor, Construction of universal bundles, II, Ann. Math. 63(3) (1956), 430-436. MR 17:1120a

25. S. B. Nadler, Jr., Hyperspaces of sets, Marcel Dekker, Inc., New York and Basel, 1978. MR 58:18330

26. R. Palais, The classification of G-spaces, Memoirs of the Amer. Math. Soc. 36, Providence, RI, 1960.

27. E. H. Spanier, Algebraic Topology, McGraw-Hill, New York, 1966. MR 35:1007

28. H. Toruńczyk, On CE-images of the Hilbert cube and characterization of $Q$-manifolds, Fund. Math. 106 (1980), 31-40. MR 83g:57006

29. H. Toruńczyk and J. E. West, The fine structure of $S^{1} / S^{1}$; a $Q$-manifold hyperspace localization of the integers, in: Proc. Internat. Conf. Geom. Topol., 439-449, PWN-Pol. Sci. Publ., Warszawa, 1980. MR 83g:57005

30. J. de Vries, Topics in the theory of topological transformation groups, in: Topological Structures II, pp. 291-304, Math. Centre Tracts, Vol. 116, Math. Centrum, Amsterdam, 1979. MR 81g:54045

31. R. Webster Convexity, Oxford Univ. Press, Oxford, 1994. MR 98h:52001

32. J. E. West, Infinite products which are Hilbert cubes, Trans. Amer. Math. Soc. 150 (1970), 1-25. MR 42:1055

33. J. E. West, Induced involutions on Hilbert cube hyperspaces, Topology Proc. 1 (1976), 281293. MR 58:24276

34. J. E. West, Open problems in infinite-dimensional topology, in: Open Problems in Topology (ed. by J. van Mill and G. Reed), 524-586, North Holland, Amsterdam-New York-OxfordTokyo, 1990. MR 92c:54001

35. M. Wojdyslawski, Rétractes absolus et hyperespaces des continus, Fund. Math. 32 (1939), 184-192.

36. R. Y. T. Wong, Noncompact Hilbert cube manifolds, preprint.

Departamento de Matematicas, Facultad de Ciencias, UnAm, Ciudad Universitaria, MÉXICO D.F. 04510, MÉXICO

E-mail address: antonyan@servidor.unam.mx 